\title{
Iterated Mayer Expansion for Classical Gases at Low Temperatures
}

\author{
Markus Göpfert and Gerhard Mack \\ II. Institut fur Theoretische Physik der Universität Hamburg, Luruper Chaussee 149, \\ D-2000 Hamburg 50, Federal Republic of Germany
}

\begin{abstract}
We derive iterated Mayer expansions for classical gases and establish recursive bounds which control their convergence. These bounds are useful for gases with two body forces which are strong and possibly attractive at distances that are short compared to their range. Our procedure is based on splitting the potential into pieces of decreasing strength and increasing range. This may be called a renormalization group treatment of a classical gas. We apply our results to Yukawa lattice gas models and obtain convergence of series expansions for the pressure for a range of parameters (temperature, fugacities, range of the interaction) that was inaccessible before. Application to 3-dimensional $U(1)$ lattice gauge theory (Coulomb gas, $Z$-ferromagnet) will be made elsewhere.
\end{abstract}

\section{Introduction}

In this paper we present some tools for the investigation of convergence properties of (generalized) Mayer expansions for classical gases [1]. They are useful when the particles of the gas interact through two body forces that are strong and possibly attractive at distances that are short compared to their range.

To be specific, consider a gas of particles with charge $m= \pm 1$ which can occupy the sites $x$ of a cubic 3 -dimensional lattice $\Lambda$ with lattice spacing set equal to 1 , and which interact through a Yukawa potential (=infrared cutoff Coulomb potential) of range $M^{-1}$. The Yukawa potential $v(x y)=\left(-\Delta+M^{2}\right)^{-1}(x y)$ is the translation invariant solution of the finite difference equation on $\mathbf{Z}^{3}$,

$$
\left(-\Delta+M^{2}\right) v(x y)=\delta_{x y} .
$$

$\Delta$ is the lattice Laplacian, viz. $\Delta f(x)=\sum_{y}(f(y)-f(x)$ ) (sum over nearest neighbours of $x$ in $\mathbf{Z}^{3}$ ).

* Work supported in part by Deutsche Forschungsgemeinschaft 
The grand canonical partition function reads

$$
Z_{\Lambda}=\sum_{N=0,1, \ldots} \frac{\bar{z}^{N}}{N !} \sum_{\substack{m_{1}, 1_{1} m_{N}, x_{N} \\\left(x_{i} \in A\right)}} \exp \left[-\frac{\beta}{2} \sum_{1 \leqq i, j \leqq N} m_{i} \nu\left(x_{i} x_{j}\right) m_{j}\right] .
$$

We have included the selfinteraction of the particles in the potential energy. Of course one can omit it and compensate for this by substituting $z$ for $\bar{z}$ on the right hand side,

$$
z=\bar{z} \exp [-\beta v(0) / 2] .
$$

Since $\left(-\Delta+M^{2}\right)^{-1}$ is a positive operator, the interaction is stable in the sense that [1]

$$
\begin{gathered}
\sum_{1 \leqq i<j \leqq N} m_{i} v\left(x_{i} x_{j}\right) m_{j} \geqq-B N, \\
B=\frac{1}{2} \vartheta(0) \equiv \frac{1}{2} \vartheta(x x) .
\end{gathered}
$$

The lattice provides a short distance cutoff to the interaction so that $v(0)<\infty$. Some such cutoff is necessary, otherwise the system would collapse.

The Mayer expansion exhibits the pressure as a power series in the fugacity

$$
\frac{1}{|\Lambda|} \ln Z_{\Lambda}=\sum_{n=1,2, \ldots} b_{n} z^{n}
$$

Stability and finite range of the interaction assure convergence of the Mayer series (1.6) for sufficiently small $z$, depending on $\beta$, uniformly in the volume $|\Lambda|$.

Estimates for $b_{n}$ can be found in $[1,2]$. Here we are interested in the behavior for large $\beta$. The best known estimates that we are aware of are in this case furnished by the recent work of Brydges and Federbush [2]

$$
\begin{gathered}
\left|b_{n}\right| \leqq(2 \beta \tilde{v}(0))^{n-1} e^{n \beta B} \frac{2}{n} e^{n-1}, \\
\tilde{v}(0) \equiv \sum_{x \in \mathbf{Z}^{3}}|\nu(x 0)|=M^{-2} .
\end{gathered}
$$

From this one obtains absolute convergence of the Mayer series (1.6) for

$$
|\bar{z}| \leqq\left(2 e \beta M^{-2}\right)^{-1} \text {. }
$$

The present investigation was carried out to prepare the ground for a rigorous proof that 3-dimensional pure $U(1)$ lattice gauge theory (with Villain action) confines static quarks for all values of the coupling parameter $\beta[3,4]$. (Details of this application will be presented elsewhere [5].) There exists an exact transformation of this system into a Coulomb gas [4]. For a sufficiently dilute Coulomb gas on a lattice, Debye screening was proven by Brydges [6], using convergence of the Mayer expansion in the presence of an infrared cutoff $M$ in the domain (1.9). To cover the case of the 3-dimensional $U(1)$ lattice gauge theory, one would want to use the Mayer expansion or a substitute for it for $\beta$ large, $\bar{z} \approx 1$, and $M$ of the order of the inverse Debye screening length $m_{D}$ (=inverse correlation length 
in the Coulomb gas in Debye-Hückel approximation). It is given by Eq. (1.18) below, with $\zeta=1$. For $\bar{z} \leqq 1$

$$
m_{D}^{2} \approx 2 \bar{z} \beta e^{-\beta v_{C b}(0) / 2}
$$

$\left[v_{C b}(0)=v(0)\right.$ at $\left.M=0\right]$. It is evident that the sufficient condition (1.9) for the convergence of the Mayer expansion is not nearly good enough to cover this case. Indeed, if $\bar{z}=1$ and $\beta \geqq 1$ it would require an infrared cutoff length $M^{-1}$ less than one lattice spacing, whereas $m_{D}^{-1}$ increases exponentially with $\beta$. In the present paper we will prove the convergence of expansions for the pressure in the desired range of parameters as described above.

It is instructive to inspect the derivation of the estimate (1.7) for the simplest coefficient $b_{2}$. This will reveal a basic reason why it is inefficient when $\beta$ is large and $M$ is small. One has the explicit formula

$$
\begin{aligned}
|\Lambda| b_{2} & =\frac{1}{2 !} \sum_{m_{1} m_{2}} \sum_{x_{1} x_{2}}\left(\exp \left[-\beta m_{1} v\left(x_{1} x_{2}\right) m_{2}\right]-1\right) \\
& =-\frac{1}{2} \beta \sum_{m_{1} m_{2}} \sum_{x_{1} x_{2}} \int_{0}^{1} d s m_{1} v\left(x_{1} x_{2}\right) m_{2} \exp \left[-\beta s m_{1} v\left(x_{1} x_{2}\right) m_{2}\right] .
\end{aligned}
$$

Now one estimates the exponential by $\exp (2 \beta B)$, using inequality (1.4). After that the $s$-integration is trivial and one obtains $\left|b_{2}\right| \leqq 2 \beta \tilde{v}(0) e^{2 \beta B}$. Bounding the exponential factor by its absolute maximum one ignores the fact that the interaction is strong (and possibly attractive) only at short distances, and not over its whole range $M^{-1}$. Another feature of the system which can be exploited is that a collection of particles at a given site with total charge zero does not interact with the rest of the system.

Our treatment of the problem is based on splitting the two body potential $v$ into pieces $v^{r}$ of increasing range and decreasing strength.

$$
v=\sum v^{r} \text {. }
$$

and treating the effect of the interactions $v^{r}$ one by one, short range interactions first. In each step a cluster expansion is performed by writing $e^{-\beta v^{r}}=1+f^{r}$ and expanding in products of $f$ 's. This may be called a renormalization group treatment [7] of the gas. ${ }^{1}$

To obtain the result for Yukawa gases mentioned above it suffices to split into two or three pieces, but the last step in this application is as difficult as a general step. We will therefore derive general recursion relations and recursive bounds first. We will then apply these to Yukawa gases with partition functions of the following from

$$
Z_{\Lambda}=\sum_{m \in Z^{\Lambda}}\left\{\prod_{x \in \Lambda} \lambda(m(x))\right\} \exp \left[-\frac{\beta}{2} \sum_{x \in \Lambda} \sum_{y \in \Lambda} m(x) v(x, y) m(y)\right]
$$

1 This becomes clearer if we transform the problem into field theoretic language by using the formula for the characteristic function of a Gaussian measure with covariance $\beta v: e^{-\beta(m, v m) / 2}$ $=\int d \mu_{\beta v}(\Phi) e^{i(m, \Phi)}$. With the split $v=\sum v^{r}$ one may introduce fields $\Phi_{r}$ whose propagators $v^{r}$ emphasize frequencies in a different range. Then one after another these fields are integrated out, high frequency parts first, by using cluster expansions 
with $\lambda(q) \geqq 0$. Summation over $m$ is over all integer valued functions on $\Lambda$. The following special models [8] can be treated simultaneously:

1) the standard gas

$$
\lambda(0)=1, \quad \lambda(q)=\zeta I_{|q|}(2 \bar{z}) / I_{0}(2 \bar{z}) \quad \text { for } \quad q= \pm 1, \pm 2, \ldots,
$$

2) the villain gas

$$
\lambda(0)=1, \quad \lambda(q)=\zeta \quad \text { for } \quad q= \pm 1, \pm 2, \ldots,
$$

3) the hard core gas

$$
\lambda(0)=1, \quad \lambda( \pm 1)=\zeta, \quad \lambda(q)=0 \quad \text { for } \quad|q|>1 .
$$

$I_{q}(\cdot)$ is the modified Bessel function. A simple combinatorial argument shows [8] that the partition function for the standard gas with $\zeta=1$ equals the partition function (1.2) times $I_{0}(2 \bar{z})^{-|\Lambda|}$. Brydges' work was concerned with this model. The Villain gas with $\zeta=1$ is the exact transform of the $U(1)$ lattice gauge theory with Villain action [9] in three dimensions. In order not to have to distinguish between special cases we set

$$
\bar{z}=1 \text { for hard core and Villain gas. }
$$

We (re)interpret $Z_{A}$ as partition functions of a gas of particles with a hard core that prevents two of them from occupying the same lattice site. They can exist in two or infinitely many states labelled by charge $q= \pm 1$ (hard core gas) and $q= \pm 1, \pm 2, \pm 3, \ldots$ (standard and Villain gas). They have a common fugacity $\zeta$, and some charge dependent selfinteraction in addition to the Yukawa interaction between different particles. We are interested in low temperatures $\beta^{-1}$ and long range $M^{-1}$ of the interaction.

Our results can be summarized in the following.

Theorem 1. There exist constants $M_{0}>0, C_{1}>0, C_{2}>0$ and $\varepsilon_{1}>0$ such that the pressure in the Yukawa gas models (1.14) admits a power series expansion in the fugacity,

$$
\frac{1}{|\Lambda|} \ln Z_{\Lambda}=\sum_{n=1,2, \ldots} b_{n} \zeta^{n}
$$

which converges absolutely and uniformly in the volume $|\Lambda|$ whenever $\beta$ is sufficiently large, $\bar{z} \geqq 0$,

$$
M_{0} \geqq M \geqq C_{1} m_{D}, \quad \text { and } \quad 1+\bar{z}(1+2|\zeta|) \leqq C_{2} \beta^{-3} e^{\beta \varepsilon_{1}} .
$$

$m_{D}^{-1}$ is the screening length in the corresponding Coulomb gas model $(M=0)$ in Debye-Hückel approximation (if $\zeta>0$ ), viz.

$$
m_{D}^{2}=2 \beta|\lambda(1)| e^{-\beta v_{C b}(0) / 2} .
$$

We note that the case $\bar{z}=\zeta=1$ is covered by this theorem, for sufficiently large $\beta$. The constant $\varepsilon_{1}$ is the same constant that will appear in Proposition 8 .

An "iterated cluster formula" for the coefficients $b_{n}$ in expansion (1.16) is given in Eq. (2.31) at the end of Sect.2. The vertex functions $\sigma$ which appear in this 
equation are recursively defined by Eq. (2.7) and (2.11). The reader should study the beginning of Sect. 2 up to Eq. (2.11) to get acquainted with the notation. Alternative formulae for the vertex functions $\sigma$ are given in Sect. 3, Eqs. (3.2) and (3.7). In Sect. 4 recursive bounds on sums of such vertex functions are derived. In Sect. 5 they are applied to the Yukawa gas models (1.14).

\section{Setup of the Expansion}

We start by rewriting the partition function (1.13) in grand canonical form in accordance with the discussion after Eq. (1.15). We introduce abbreviations

$$
\begin{gathered}
\xi_{i}=\left(m_{i}, x_{i}\right) ; \quad \int d \xi_{i}(\ldots)=\sum_{m_{i}= \pm 1, \pm 2, \ldots} \sum_{x_{i} \in \Lambda}(\ldots), \\
v\left(\xi_{i}, \xi_{j}\right)= \begin{cases}+\infty & \text { if } x_{i}=x_{j} \\
m_{i} m_{j} v\left(x_{i}, x_{j}\right) & \text { otherwise }\end{cases}
\end{gathered}
$$

In this notation

$$
\begin{aligned}
& Z_{\Lambda}=\sum_{N=0,1, \ldots} Z_{\Lambda}^{N}=\sum_{N=0,1, \ldots} \int d \xi_{1} \ldots d \xi_{N} \mathscr{Z}^{N}\left(\xi_{1} \ldots \xi_{N}\right), \\
& \mathscr{Z}^{N}\left(\xi_{1} \ldots \xi_{N}\right)=\frac{1}{N !}\left\{\prod_{i=1}^{N} \lambda\left(m_{i}\right)\right\} \exp \left[-\frac{\beta}{2} \sum_{i, j=1}^{N} v\left(\xi_{i}, \xi_{j}\right)\right] .
\end{aligned}
$$

$Z_{A}^{N}$ is proportional to $\zeta^{N}$.

We shall split the interaction potential into $R$ pieces of increasing range and decreasing strength.

$$
v\left(\xi_{i}, \xi_{j}\right)=v^{0}\left(\xi_{i}, \xi_{j}\right)+\sum_{r=1}^{R-1} v^{r}\left(\xi_{i}, \xi_{j}\right)
$$

$v^{0}$ incorporates the hard core

$$
v^{0}\left(\xi_{i}, \xi_{j}\right)= \begin{cases}+\infty & \text { if } x_{i}=x_{j}, \quad i \neq j \\ 0 & \text { otherwise } .\end{cases}
$$

The other pieces of the interaction shall assume finite values only. They remain arbitrary until we come to doing estimates.

We write down an iterated cluster expansion. We consider clusters of clusters of $\ldots$ of clusters of particles $=$ constituents. They will be called $\ell$-vertices, $\ell=0,1 \ldots R$. A 0 -vertex is a single particle $i$ which is its own constituent. Associated with it is a variable $\xi_{i}=\left(m_{i}, x_{i}\right)$ which specifies its state and position, and a vertex function

$$
\sigma^{0}\left(\xi_{i}\right)=\lambda\left(m_{i}\right)
$$

Higher vertices are defined inductively. An $\ell$-vertex $\alpha^{\prime}$ is a finite collection $\{\alpha\}$ of $(\ell-1)$-vertices, no two of which share a constituent. There is an associated variable

$$
\xi_{\alpha^{\prime}}=\left(\left\{\xi_{\alpha}\right\}_{\alpha \in \alpha^{\prime}}\right) ; \quad d \xi_{\alpha^{\prime}}=\prod_{\alpha \in \alpha^{\prime}} d \xi_{\alpha}
$$


A particle $i$ is constituent of $\alpha^{\prime}$ if it is constituent of one of the $(\ell-1)$-vertices $\alpha \in \alpha^{\prime}$. We write $i € \alpha$ in this case, and $C\left(\alpha^{\prime}\right)$ for the set of all constituents of $\alpha^{\prime}$. We use the symbol $\sum$ for union of sets with vanishing mutual intersection.

We introduce the type $\left[\alpha^{\prime}\right]$ of a $\ell$-vertex. $\left[\alpha^{\prime}\right]$ is an equivalence class of $\ell$ vertices. All 0 -vertices are equivalent, so there is only one type of 0 -vertex. Two $\ell$ vertices belong to the same equivalence class if they contain the same number of $(\ell-1)$-vertices of each type $[\alpha]$. Thus, for instance, two 1 -vertices belong to the same class if they contain the same number of constituents. We write $T_{\ell}$ for the set of all types of $\ell$-vertices. Two collections $\left\{\alpha^{\prime}\right\}$ of $\ell$-vertices are said to be of the same type $\left\{\left[\alpha^{\prime}\right]\right\}$ if both contain equally many $\ell$-vertices of each type $\left[\gamma^{\prime}\right] \in T_{\ell^{\prime}}$.

It is convenient to introduce an abbreviation for interactions. If $\alpha, \gamma$ are two $\ell$ vertices we write

$$
\begin{aligned}
& v^{\ell}(\alpha \gamma)=\sum_{i \in \alpha} \sum_{j \in \beta} v^{\ell}\left(\xi_{i}, \xi_{j}\right) \text { for } \alpha \neq \gamma \\
& v^{\ell}(\alpha \alpha)=\frac{1}{2} \sum_{i, j \in \alpha} v^{\ell}\left(\xi_{i}, \xi_{j}\right) .
\end{aligned}
$$

$v^{\ell}(\alpha \alpha)$ includes in particular the self interaction of all constituents of the $\ell$-cluster $\alpha$ that is due to the piece $v^{\ell}$ in the potential. We also introduce the quantity $f_{\alpha \beta}^{\ell}$ for distinct $\ell$-vertices $\alpha \neq \beta$. It depends on $\xi_{\alpha}$ and $\xi_{\beta}$ and is given by

$$
1+f_{\alpha \gamma}^{\ell}=\exp \left[-\beta v^{\ell}(\alpha \gamma)\right] \text {. }
$$

Now we will write down a recursion formula which defines the vertex functions inductively. We write $\left\{\mathscr{G}_{\alpha^{\prime}}\right\}$ for the set of all connected graphs with vertices $\alpha \in \alpha^{\prime}$. Such a graph is specified by a set of links $=$ unordered pairs $(\alpha, \beta)$, with $\alpha \neq \beta$ and $\alpha, \beta \in \alpha^{\prime}$. We define

$$
\sigma_{\alpha^{\prime}}^{l+1}=\left\{\prod_{[\beta] \in T_{\ell}} \frac{1}{\left.N_{[\beta]}^{\alpha^{\prime}}\right\}}\right\} \sum_{\mathscr{G}_{\alpha^{\prime}}}\left\{\left(\prod_{\alpha \in \alpha^{\prime}} \sigma_{\alpha}^{\ell} e^{-\beta v^{\ell}(\alpha \alpha)}\right)\left(\prod_{(\alpha \gamma) \in \mathscr{G}_{\alpha^{\prime}}} f_{\alpha \gamma}^{\ell}\right)\right\} .
$$

It depends on $\xi_{\alpha^{\prime}}$. Here $N_{[\beta]}^{\alpha^{\prime}}$ is the number of $\ell$-vertices of type $[\beta]$ in the $(\ell+1)$ vertex $\alpha^{\prime}$.

We claim that the Boltzmann factor $\mathscr{Z}^{N}\left(\xi_{1} \ldots \xi_{N}\right)$ may be expressed in terms of these vertex functions as follows

$$
\begin{aligned}
\mathscr{Z}^{N}\left(\xi_{1} \ldots \xi_{N}\right)= & \sum_{\Sigma C(\alpha)}^{\left.\sum_{\{\alpha\}}^{\prime} \ldots N\right\}} \frac{1}{N !} K^{\ell}(\{\alpha\}) \mathbf{S}^{(c)}\left\{\left[\prod_{\alpha \in\{\alpha\}} \sigma_{\alpha}^{\ell}\left(\xi_{\alpha}\right)\right]\right. \\
& \left.\cdot \exp \left[-\frac{\beta}{2} \sum_{\alpha, \gamma \in\{\alpha\}} V^{\ell}(\alpha \gamma)\right]\right\} .
\end{aligned}
$$

$\mathbf{S}^{(c)}$ is an average over all $N$ ! permutations of the $N$ constituents $i=1 \ldots N . K^{\ell}(\{\alpha\})$ are combinatorial factors which will be determined below; the result is given in Eq. (2.25). Summation is over collections of $\ell$-vertices $\alpha$ which share no constituent and have a total of $N$ constituents $i=1 \ldots N$. Only one representative $\{\alpha\}$ out of every collection $\{[\alpha]\}$ of types of $\ell$-vertices is to be included in the sum. This is indicated by the prime'. This restriction could of course be dropped, but the 
combinatorial factor would then be different. The last factor in (2.12) involves the residual interaction not yet incorporated into $\sigma^{\ell}$, viz.

$$
\begin{gathered}
V^{\ell}(\alpha \gamma)=\sum_{i \leqq \alpha} \sum_{j \leqq \gamma} \sum_{r \geqq \ell} v^{r}\left(\xi_{i}, \xi_{j}\right) \text { for } \alpha \neq \gamma, \\
V^{\ell}(\alpha \alpha)=\sum_{i, j \leqq \alpha} \sum_{r \geqq \ell} v^{r}\left(\xi_{i}, \xi_{j}\right) .
\end{gathered}
$$

The proof of Eq. (2.12) proceeds by induction.

$\ell=0$ : In this case $\alpha, \gamma$ are individual particles. Since there is only one type of 0 vertex, the sum over $\{\alpha\}$ in (2.12) has only one term. If we insert Eq. (2.7) for $\sigma^{0}$ and compare Eqs. (2.13), (2.14) with (2.5) we see that Eq. (2.12) agrees with (2.4) with

$$
K^{0}(\{\alpha\})=1 \text {. }
$$

The symmetrizer $\mathbf{S}^{(c)}$ in (2.12) is redundant if $\ell=0$.

Induction Step: Suppose that Eq. (2.12) is true for a given $\ell \geqq 0$. We show its validity for $\ell+1$. We split

$$
V^{\ell}(\alpha \gamma)=v^{\ell}(\alpha \gamma)+\sum_{r \geqq \ell+1}\left\{\sum_{i \leqq \alpha} \sum_{j \subseteq \gamma} v^{r}\left(\xi_{i}, \xi_{j}\right)\right\} \text { for } \alpha \neq \gamma
$$

and similarly for $\alpha=\gamma$. Using definition (2.10) we can then write

$$
\begin{aligned}
\exp \left[-\frac{\beta}{2} \sum_{\alpha, \gamma} V^{\ell}(\alpha \gamma)\right]= & \left\{\prod_{\alpha} e^{-\beta v^{\ell}(\alpha \alpha)}\right\}\left\{\prod_{(\alpha \gamma)}\left[1+f_{\alpha \gamma}^{\ell}\right]\right\} . \\
& \cdot \exp \left[-\frac{\beta}{2} \sum_{i, j} \sum_{r \geqq \ell+1} v^{r}\left(\xi_{i}, \xi_{j}\right)\right] .
\end{aligned}
$$

The product in the second \{\} is over all unordered pairs of distinct $\ell$-vertices $\alpha, \gamma$. We expand this product and proceed in the standard way.

$$
\prod_{(\alpha \gamma)}\left[1+f_{\alpha \gamma}^{\ell}\right]=\sum_{\mathscr{B}} \prod_{(\alpha \gamma) \in \mathscr{B}} f_{\alpha \gamma}^{\ell}
$$

Summation is over all not necessarily connected graphs $\mathscr{B}$ with vertices in the set $\{\alpha\}$ of $\ell$-vertices that is specified by whatever term in the sum $\sum_{\{\alpha\}}^{\prime}$ in (2.12) we consider. Some of the connected components of $\mathscr{B}$ may be single $\ell$-vertices. We decompose $\mathscr{B}$ into connected graphs $\mathscr{G}_{\alpha^{\prime}}$ with vertices $\alpha \in \alpha^{\prime} . \alpha^{\prime}$ is a set of $\ell$-vertices with no common constituents and is therefore a $(\ell+1)$-vertex.

$$
\prod_{(\alpha \gamma)}\left[1+f_{\alpha \gamma}^{\ell}\right]=\sum_{\substack{\left\{\alpha^{\prime}\right\} \\ \text { compatible with }\{\alpha\}}}\left\{\prod_{\alpha^{\prime}}\left(\sum_{\mathscr{G}_{\alpha^{\prime}}}\left[\prod_{(\alpha \gamma) \in \mathscr{G}_{\alpha^{\prime}}} f_{\alpha \gamma}^{\ell}\right]\right)\right\} .
$$

Summation over $\left\{\alpha^{\prime}\right\}$ is over all sets of $(\ell+1)$-vertices which consist of distinct $\ell$ vertices in $\{\alpha\}$ and have the property that each $\ell$-vertex in $\{\alpha\}$ is in some $(\ell+1)$ vertex $\alpha^{\prime}$ (i.e. it runs over partitions of the set $\{\alpha\}$ ). 
We insert Eqs. (2.17) and (2.18) into expression (2.12) for $\mathscr{Z}^{N}$ to obtain

$$
\begin{aligned}
\mathscr{Z}^{N}\left(\xi_{1} \ldots \xi_{N}\right)= & \sum_{\substack{\{\alpha\}\\
}} \frac{1}{N !} K^{\ell}(\{\alpha\}) \\
& \cdot \mathbf{S}^{(c)} \sum_{\substack{\left\{\alpha^{\prime}\right\} \\
\text { compatible with }\{\alpha\}}}\left\{\prod_{\alpha^{\prime}} \sum_{\mathscr{G}_{\alpha^{\prime}}}\left(\prod_{\alpha \in \alpha^{\prime}} \sigma_{\alpha}^{\ell} e^{-\beta v^{\ell}(\alpha \alpha)}\right) \prod_{(\alpha \gamma) \in \mathscr{G}_{\alpha^{\prime}}} f_{\alpha \gamma}^{\ell}\right\} \\
& \cdot \exp \left[-\frac{\beta}{2} \sum_{i, j} \sum_{r \geqq \ell+1} v^{r}\left(\xi_{i}, \xi_{j}\right)\right]
\end{aligned}
$$

We inspect the last factor. Comparing with definitions (2.13), (2.14) we see that

$$
\frac{1}{2} \sum_{i, j} \sum_{r \geqq \ell+1} v^{r}\left(\xi_{i} \xi_{j}\right)=\frac{1}{2} \sum_{\alpha^{\prime}, \gamma^{\prime}} V^{\ell+1}\left(\alpha^{\prime} \gamma^{\prime}\right)
$$

We may now insert definition (2.11) of the vertex functions to rewrite expression (2.19) as

$$
\begin{aligned}
\mathscr{Z}^{N}\left(\xi_{1} \ldots \xi_{N}\right)= & \sum_{\left.\sum C(\alpha) \stackrel{\{\alpha\}}{=}{ }^{\prime} \ldots N\right)} \frac{1}{\mathrm{~N} !} K^{\ell}(\{\alpha\}) \\
& \cdot \mathbf{S}^{(c)} \sum_{\substack{\left\{\alpha^{\prime}\right\} \\
\text { compatible with }\{\alpha\}}}\left\{\left[\prod_{\alpha^{\prime} \in\left\{\alpha^{\prime}\right\}}\left(\sigma_{\alpha^{\prime}}^{\ell+1} \prod_{[\beta] \in T_{\ell}} N_{[\beta]}^{\alpha^{\prime} !}\right)\right]\right. \\
& \left.\cdot \exp \left[-\frac{\beta}{2} \sum_{\alpha^{\prime}, \gamma^{\prime} \in\left\{\alpha^{\prime}\right\}} V^{\ell+1}\left(\alpha^{\prime} \gamma^{\prime}\right)\right]\right\} .
\end{aligned}
$$

We are left with a combinatorial problem. Any two representatives $\left\{\alpha^{\prime}\right\}$ of the same equivalence class $\left\{\left[\alpha^{\prime}\right]\right\}$ that is specified by a collection of types of $(\ell+1)$ vertices differ by some permutation of constituents. Because of the presence of the symmetrizer $\mathbf{S}^{(c)}$ they will give the same contribution to expression (2.20). Therefore, expression (2.20) is of the form (2.12) with $\ell+1$ substituted for $\ell$, and

$$
\begin{gathered}
K^{\ell+1}\left(\left\{\alpha^{\prime}\right\}\right)=K^{\ell}(\{\alpha\}) n^{\ell}\left(\left\{\alpha^{\prime}\right\}\right) \prod_{\alpha^{\prime} \in\left\{\alpha^{\prime}\right\}} \prod_{[\beta] \in T_{\ell}} N_{[\beta]}^{\alpha^{\prime}} ! \\
\{\alpha\} \equiv\left\{\alpha \in \alpha^{\prime}, \alpha \in\left\{\alpha^{\prime}\right\}\right\} .
\end{gathered}
$$

$n^{\ell}\left(\left\{\alpha^{\prime}\right\}\right)$ is the number of collections of $(\ell+1)$-vertices of type $\left\{\left[\alpha^{\prime}\right]\right\}$ that can be made out of a given set of $\ell$-vertices $\alpha \in \alpha^{\prime}$. This completes the proof of Eq. (2.12).

It remains to determine the combinatorial factor $n^{\ell}\left(\left\{\alpha^{\prime}\right\}\right)$ and to solve the recursion relation (2.21) with initial condition (2.15) for the combinatorial factors $K^{\ell}(\{\alpha\})$. The reader is invited to skip the following discussion and continue with Eq. (2.25).

To facilitate visualization, $\ell$-vertices will be simply called vertices in the following discussion, and $(\ell+1)$-vertices will be called clusters. Vertices can be of different type $=$ colour.

In Fig. 1, vertices of different colour are distinguished by symbols $\square, \nabla, \bigcirc, \times$. Consider a set of labelled vertices, possibly of different colours. We partition this set into clusters $\alpha^{\prime}$. Two clusters are of the same type if both contain an equal 


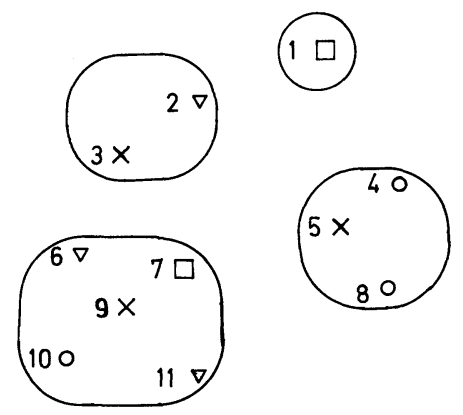

Fig. 1. Clusters of labelled vertices of different colour

number of vertices for each colour. Two partitions $\left\{\alpha^{\prime}\right\}$ are of the same type if both contain the same number of clusters of each type. Two partitions $\left\{\alpha^{\prime}\right\}$ of the same type are obtained from each other by permutation of vertices of the same colour $[\beta]$. Permutation of vertices of a given colour within a cluster does not produce a new partition, and neither does a permutation of vertices which is equivalent to a permutation of clusters of equal type. Thus the number $n^{\ell}\left(\left\{\alpha^{\prime}\right\}\right)$ of partitions of the same type is

$$
n^{\ell}\left(\left\{\alpha^{\prime}\right\}\right)=\frac{\prod_{[\beta] \in T_{\ell}} N_{[\beta]}^{\ell !}}{\left(\prod_{\alpha^{\prime}} \prod_{[\beta] \in T_{\ell}} N_{[\beta]}^{\alpha^{\prime} !}\right)_{\left[\gamma^{\prime}\right] \in T_{\ell+1}} N_{\left[\gamma^{\prime}\right]}^{\ell+1 !}} .
$$

$T_{\ell}$ is the set of types of $\ell$-vertices (colours), $T_{\ell+1}$ is the set of types of $(\ell+1)$-vertices (clusters), $N_{\left[\gamma^{\prime}\right]}^{\ell+1}$ is the number of $(\ell+1)$-vertices of type $\left[\gamma^{\prime}\right] \in T_{\ell+1}$ in the collection $\left\{\alpha^{\prime}\right\}$ of $(\ell+1)$-vertices, and $N_{[\beta]}^{\ell}=\sum_{\alpha^{\prime} \in\left\{\alpha^{\prime}\right\}} N_{[\beta]}^{\alpha^{\prime}}$ is the number of $\ell$-vertices of type $[\beta] \in T_{\ell}$ in the collection $\{\alpha\}$ of $\ell$-vertices that is specified by $\left\{\alpha^{\prime}\right\}$ according to Eq. (2.22).

We can insert result (2.23) into recursion relation (2.21) to obtain

$$
K^{\ell+1}\left(\left\{\alpha^{\prime}\right\}\right)=K^{\ell}(\{\alpha\}) \frac{\prod_{[\beta] \in T_{\ell}} N_{[\beta]}^{\ell} !}{\prod_{\left[\beta^{\prime}\right] \in T_{\ell+1}} N_{\left[\beta^{\prime}\right]}^{\ell+1} !}
$$

In the special case $\ell=0$ there is only one type of 0 -vertex $[\beta]$, and $N_{[\beta]}^{0}=N=$ number of constituents in $\{\alpha\}$. The solution of the recursion relation (2.24) with initial condition (2.15) is therefore

$$
\frac{1}{N !} K^{\ell}(\{\alpha\})=\frac{1}{\prod_{[\beta] \in T_{\ell}} N_{[\beta]}^{\ell} !} .
$$

$N_{[\beta]}^{\ell}=$ number of $\ell$-vertices of type $[\beta]$ in the collection $\{\alpha\}$ of $\ell$-vertices. This completes the discussion of combinatorial factors. 
Let us return to expression (2.12) for the Boltzmann factor and specialize to $\ell=R$. From the definition (2.13), (2.14) of $V^{\ell}$ and Eq. (2.5) it follows that

$$
V^{R}=0 \text {. }
$$

Therefore we obtain the following proposition as a special case of Eq. (2.12).

Proposition 2. The Boltzmann factor (2.4) can be expressed in terms of the vertex functions as follows

$$
\mathscr{Z}^{N}\left(\xi_{1} \ldots \xi_{N}\right)=\sum_{\Sigma C(\alpha)=\{1 \ldots N\}}^{\prime}\left(\prod_{[\beta] \in T_{R}} N_{[\beta]}^{R} !\right)^{-1} \mathbf{S}^{(c)} \prod_{\alpha \in\{\alpha\}} \sigma_{\alpha}^{R}\left(\xi_{\alpha}\right) .
$$

Notation and terminology were explained at the beginning of this section (up to Eq. (2.11)). Summation is over collections of $R$-vertices $\alpha$ which share no constituent and have a total of $N$ constituents $i=1 \ldots N$. Only one representative $\{\alpha\}$ out of every collection $\{[\alpha]\}$ of types of $R$-vertices is to be included in the sum. This is indicated by the prime'. The symmetrizer $\mathbf{S}^{(c)}$ averages over all $N$ ! permutations of the $N$ constituents $i=1 \ldots N . N_{[\beta]}^{R}$ is the number of $R$-vertices of type $[\beta]$ in $\{\alpha\}$.

$\mathrm{Up}$ to this point all our manipulations involved expansions in finite sums and no question of convergence arose. In the following discussion it will be assumed that

$$
\sum_{[\alpha] \in T_{R}} \int d \xi_{\alpha}\left|\sigma_{\alpha}^{R}\left(\xi_{\alpha}\right)\right|<\infty
$$

This implies in particular that the $\xi$-summations in the individual terms in the sum over $[\beta]$ are absolutely convergent. We may therefore sum expressions (2.26) to obtain the canonical partition functions

$$
Z_{\Lambda}^{N}=\sum_{\Sigma C(\alpha)=\{1 \ldots N\}}^{\sum_{\{\alpha\}}^{\prime}}\left(\prod_{[\beta] \in T_{R}} N_{[\beta]}^{R} !\right)^{-1} \prod_{\alpha \in\{\alpha\}}\left\{\int d \xi_{\alpha} \sigma_{\alpha}^{R}\left(\xi_{\alpha}\right)\right\} .
$$

Summation is over one representative out of each collection of types $\{[\alpha]\}$ and is therefore equivalent to a sum over over multiplicities $N_{[\beta]}^{R}$ with which $R$-vertices of type $[\beta] \in T_{R}$ appear. The expression in \{\} depends only on the type $[\alpha]$ of $\alpha$. Thus

$$
Z_{\Lambda}^{N}=\sum_{\substack{\left\{N_{[\beta]}^{R}\right\}\{\beta] \in T_{R} \\ \sum[\beta]^{N}[\beta]}} \prod_{[\gamma] \in T_{R}}\left\{\frac{1}{N_{[\gamma]}^{R} !}\left[\int d \xi_{\gamma} \sigma_{\gamma}^{R}\left(\xi_{\gamma}\right)\right]^{\left.N_{[\gamma]}^{R}\right]}\right\} .
$$

The constraint on the sum says that the total number of constituents is $N$. If we insert Eq. (2.29) into the definition (2.3) of the grand canonical function, this constraint disappears and the sums factorize [10]. The result is given by the following

$$
\text { Proposition 3. } \quad Z_{\Lambda}=\exp \sum_{[\alpha] \in T_{R}} \int d \xi_{\alpha} \sigma_{\alpha}^{R}\left(\xi_{\alpha}\right),
$$

provided the sum in the exponent is absolutely convergent, i.e. inequality (2.27) holds. The series in the exponent is of the form (1.16). 
The (rigorous) derivation of Eq. (2.30) from (2.29), (2.27) is standard; details can be found in Appendix A.

It follows from their definition that $\sigma_{\gamma}^{\ell}$ are proportional to $\zeta^{|C(\gamma)|}$, where $|C(\gamma)|=$ number of constituents in the $\ell$-vertex $\gamma$. Therefore the exponent in $(2.30)$ is a power series expansion in $\zeta$ and represents an analytic function of $\zeta$ if it is absolutely convergent. It can be rewritten in the form (1.16) with

$$
|\Lambda| b_{n}=\sum_{\substack{[\gamma] \in T_{R} \\|\mathcal{C}(\gamma)|=n}} \int d \xi_{\gamma} \sigma_{\gamma}^{R}\left(\xi_{\gamma}\right) .
$$

$\int d \xi_{\gamma}$ stands for summation over charge and location of the $n$ particles which are the constituents of the $R$-vertex $\gamma$.

\section{The Tree Formula}

In this section we will present alternative formulae for the vertex functions $\sigma_{\alpha}^{\ell}$. We start with 1-vertices. The 1-vertex functions are determined by the properties of the hard core potential $v^{0}$, Eq. (2.6), alone. To determine them we may therefore ignore the rest of the potential and set $R=1$.

Consider a lattice gas of particles which can exist in several states $q$, with (sufficiently small) fugacities $\lambda(q)$ that may depend on $q$, and no interaction except a hard core that prevents two particles from occupying the same lattice site. The partition function for such a gas is equal to

$$
Z_{\Lambda}=\left[1+\sum_{q} \lambda(q)\right]^{|\Lambda|}
$$

Therefore the pressure is

$$
\frac{1}{|\Lambda|} \ln Z_{\Lambda}=\sum_{n \geqq 1} \frac{(-1)^{n-1}}{n}\left[\sum_{q} \lambda(q)\right]^{n}
$$

We compare this power series in variables $\lambda(q)$ with the result of setting $R=1$ in Eq. (2.30). From the definitions (2.6)...(2.11), it follows that

$$
\sigma^{1}\left(\xi_{1} \ldots \xi_{n}\right)=0, \quad \text { unless } x_{1}=x_{2} \ldots=x_{n} .
$$

The comparison therefore yields the unique result

$$
\sigma^{1}\left(\xi_{1} \ldots \xi_{n}\right)=\frac{(-1)^{n-1}}{n} \lambda\left(m_{1}\right) \ldots \lambda\left(m_{n}\right) \delta_{x_{2} x_{1}} \ldots \delta_{x_{n} x_{1}} .
$$

Now we turn to the higher vertex functions. In later estimates we will use the tree formula, Eq. (3.7) below, in place of the recursive definition (2.11) of vertex functions. The tree formalism was developed in constructive field theory [11]. The tree formula for the cluster integrals in the Mayer expansion for classical gases was derived by Brydges and Federbush [2]. It is readily generalized to our case (see Appendix B). To state the result, we recall the pertinent definitions.

Consider the set of all functions $\eta$ which assign to every integer $a=1 \ldots t-1$ a positive integer $\eta(i)$ satisfying

$$
\eta(i) \leqq i
$$




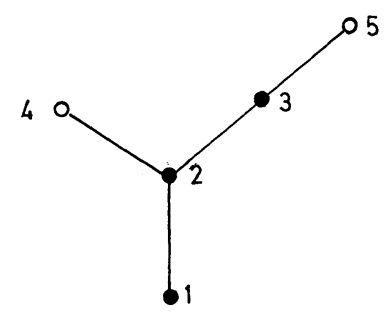

Fig. 2. A tree. Maximal vertices are indicated by open circles. They are characterized by $i \notin$ range $\eta$

$$
\eta(4)=3, \quad \eta(3)=\eta(2)=2, \quad \eta(1)=1
$$

Every such function specifies a tree graph with $t$ vertices $1 \ldots t$. Its links are the pairs $(a+1, \eta(a)), a=1 \ldots t-1$. An example is shown in Figure 2. (The numbering of the vertices is compatible with the partial ordering that is specified by the tree graph in the natural way, and every such numbered tree is obtained from a function $\eta$ as specified above.) The endpoints of the branches of the tree will be called maximal vertices.

One introduces real variables $s_{1} \ldots s_{t-1}$ which take values $0 \ldots 1$, and the functions

$$
f\left(\eta, s_{1} \ldots s_{t-1}\right)=\prod_{a=1}^{t-1} s_{a-1} s_{a-2} \ldots s_{\eta(a)} .
$$

Empty products which arise when $\eta(a)=a$ or $t=1$ are read as 1 .

Consider now an $(\ell+1)$-vertex $\alpha^{\prime}$ which consists of $t \ell$-vertices. We shall label them in some arbitrary way $\alpha_{1} \ldots \alpha_{t}$. The symbol $\mathbf{S}$ will stand for symmetrization in labels $\alpha_{1} \ldots \alpha_{t}$. It acts on symbolic expressions $F$ carrying such labels

$$
\mathbf{S} F\left(\alpha_{1} \ldots \alpha_{t}\right)=\frac{1}{t !} \sum_{\pi} F\left(\alpha_{\pi 1} \ldots \alpha_{\pi t}\right)
$$

(sum over all $t$ ! permutations of $(1 \ldots t)$ ). By definition (2.11), the vertex function $\sigma_{\alpha^{\prime}}^{\ell+1}$ is symmetric in the labels $\alpha_{1} \ldots \alpha_{t}$.

Given the potentials $v^{\ell}\left(\alpha_{i} \alpha_{j}\right)$, one defines a partially decoupled interaction $W^{\ell}$. It depends on $\mathbf{s}=\left(s_{1} \ldots s_{t-1}\right)$

$$
W^{\ell}\left(\mathbf{s} \mid \alpha^{\prime}\right)=\sum_{a=1}^{t} v^{\ell}\left(\alpha_{a} \alpha_{a}\right)+\sum_{1 \leqq a<b \leqq t} s_{a} s_{a+1} \ldots s_{b-1} v^{\ell}\left(\alpha_{a} \alpha_{b}\right) .
$$

The tree formula reads for $\ell \geqq 1$.

$$
\begin{aligned}
\sigma_{\alpha^{\prime}}^{\ell+1} \equiv \frac{t !}{\prod_{[\beta] \in T_{\ell}} N_{[\beta]}^{\alpha^{\prime}} !} \hat{\sigma}_{\alpha^{\prime}}^{\ell+1} \\
\hat{\sigma}_{\alpha^{\prime}}^{\ell+1}\left(\xi_{\alpha^{\prime}}\right)=\frac{(-\beta)^{t-1}}{t} \mathbf{S} \int_{0}^{1} d s_{1} \ldots d s_{t-1} \sum_{\eta} \\
\cdot\left\{f(\eta, \mathbf{s})\left[\prod_{a=1}^{t-1} v^{\ell}\left(\alpha_{a+1}, \alpha_{\eta(a)}\right)\right] e^{-\beta W\left(\mathbf{s} \mid \alpha^{\prime}\right)} \prod_{b=1}^{t} \sigma_{\alpha_{b}}^{\ell}\left(\xi_{\alpha_{b}}\right)\right\} .
\end{aligned}
$$

Summation is over trees as described above. 
Proposition 4. The tree formula (3.7) is equivalent to the defining Eq.(2.11) for the vertex functions, for $\ell \geqq 1$.

The proof is relegated to Appendix B. (It establishes a substitute for the decomposition (2.18). The assertion follows then by a uniqueness argument.)

To obtain bounds on vertex functions $\sigma^{\ell}$, estimates on $W^{\ell}\left(\mathbf{s} \mid \alpha^{\prime}\right)$ will be needed. They will be derived using the fact that $W^{t}$ can be exhibited as a convex combination of partially decoupled interactions as follows [2]. Suppose a potential $W_{r}$ is of the form

$$
W_{r}=\frac{1}{2} \sum_{1 \leqq a, b \leqq t} w_{r}(a, b) .
$$

Then one defines, for $n=0 \ldots t-1$, a partially decoupled potential

$$
W_{r, n}=\frac{1}{2} \sum_{1 \leqq a, b \leqq n} w_{r}(a, b)+\frac{1}{2} \sum_{n<a, b \leqq t} w_{r}(a, b) .
$$

To obtain $W^{\ell}$ we set

$$
w_{0}(a, b)=v^{\ell}\left(\alpha_{a}, \alpha_{b}\right) \text {. }
$$

The quantities $W_{r}$ for $r=1 \ldots t-1$ depend on variables $\mathbf{s}=\left(s_{1} \ldots s_{t-1}\right)$. They are recursively defined by

$$
W_{r}=\left(1-s_{r}\right) W_{r-1, r}+s_{r} W_{r-1} .
$$

In this way one constructs

$$
W^{\ell}\left(\mathbf{s} \mid \alpha^{\prime}\right)=W_{t-1}(\mathbf{s})
$$

\section{Recursive Estimates}

To get useful estimates, some bounds on the pieces $v^{\ell}$ are needed from which the potential $v$ is composed, see Eq. (2.5). We assume that $v^{\ell}$ are of the form

$$
v^{\ell}\left(\xi_{i}, \xi_{j}\right)=m_{i} m_{j} v^{\ell}\left(x_{i} x_{j}\right) \text { or } 0, \text { for } \ell \geqq 1,
$$

and that they satisfy inequalities of the form

$$
\frac{1}{2} \sum_{1 \leqq i, j \leqq N} v^{\ell}\left(\xi_{i}, \xi_{j}\right) \geqq \gamma_{\ell}+\varepsilon_{\ell}\left(-1+\sum_{i=1}^{N} m_{i}^{2}\right) \text { for } \quad N \geqq 1,
$$

with

$$
\begin{aligned}
& \varepsilon_{1}>0, \quad \varepsilon_{\ell} \geqq 0 \quad(\ell=2 \ldots R-1), \\
& \delta_{\ell} \equiv \gamma_{\ell}-\varepsilon_{\ell} \geqq 0 \quad(\ell=1 \ldots R-1) .
\end{aligned}
$$

We will show in Sect. 5 how the Yukawa potential can be split in such a way that inequalities of this form are true, with $\gamma_{1}=\frac{1}{2} v^{1}(0)$.

We begin by deriving inequalities for the vertex functions themselves. $\xi$ summations will be done afterwards. The final step will be to estimate sums over types of $\ell$-vertices. 
Consider formula (3.7) for the vertex functions $\sigma_{\alpha^{\prime}}^{\ell+1}$ for $\ell \geqq 1$. Remember that the $(\ell+1)$-vertex $\alpha^{\prime}$ consists of some number $t \geqq 1$ of $\ell$-vertices $\alpha_{1} \ldots \alpha_{t}$ that were numbered in some arbitrary way. We note first that the bounds (4.2) imply a similar bound for the partially decoupled interaction $W^{t}\left(\mathbf{s} \mid \alpha^{\prime}\right)$

$$
W^{\ell}\left(\mathbf{s} \mid \alpha^{\prime}\right) \geqq \delta_{\ell}+\varepsilon_{\ell} \sum_{j \subseteq \alpha^{\prime}} m_{j}^{2} .
$$

This is proven by noting that the inequality is preserved throughout the recursive procedure (3.10)...(3.12) by which $W^{\ell}$ can be constructed because formation of convex combinations preserves inequalities.

Inserting the bound (4.3) into formula (3.7) for $\sigma_{\alpha^{\prime}}^{\ell+1}$ and using (4.1), one obtains the inequality

$$
\begin{aligned}
\left|\hat{\sigma}_{\alpha^{\prime}}^{\ell+1}\left(\xi_{\alpha^{\prime}}\right)\right| \leqq & \frac{\beta^{t-1}}{t} e^{-\beta\left(\delta_{\ell}+\varepsilon_{\ell} \Sigma_{J \subseteq \alpha^{\prime}} m_{j}\right)}\left\{\prod_{b=1}^{t}\left|\sigma_{\alpha_{b}}^{\ell}\left(\xi_{\alpha_{b}}\right)\right|\right\} \\
& \cdot \mathbf{S} \int_{0}^{1} d s_{1} \ldots d s_{t-1} \sum_{\eta} \prod_{a=1}^{t-1} \\
& \cdot\left\{\sum_{j \in \alpha_{a}+1} \sum_{k \in \alpha_{\eta(a)}} s_{a-1} s_{a-2} \ldots s_{\eta(a)}\left|m_{j} m_{k} \nu^{\ell}\left(x_{j} x_{k}\right)\right|\right\} .
\end{aligned}
$$

To obtain $\sigma_{\alpha^{\prime}}^{\ell+1}$ one has to multiply with the combinatorial factor of Eq. (3.7a).

Next we carry out $x$-summations and do the $s$-integrations. We assume that the potentials $v^{\ell}$, and therefore also the vertex functions, are translation invariant functions of the positions of particles $x_{i} \in \mathbb{Z}^{3}$. [This entails no loss of generality. If translation invariance in this sense does not hold, the following procedure should be preceded by a preparatory step where $|\sigma|,\left|v^{\ell}\right|$ etc. are bounded above by functions which depend only on differences of arguments,

$$
\left.f\left(x_{1} \ldots x_{n}\right) \rightarrow \sup f\left(x_{1}+a \ldots x_{n}+a\right)\right] \text {. }
$$

We write $\xi_{\alpha}=\left(m_{\alpha}, x_{\alpha}\right), x_{\alpha}=\left(x_{i}\right)_{i \subseteq \alpha}$ etc., and introduce a (preliminary) norm $(n=$ number of constituents of $\alpha$ )

$$
\begin{aligned}
\left\|\sigma_{\alpha}^{\ell}\right\|\left(m_{\alpha}\right) & =\sum_{x_{\alpha} \in \mathbf{Z}^{3 n}}\left|\sigma_{\alpha}^{\ell}\left(\xi_{\alpha}\right)\right| \delta_{x_{2} x} \\
& \geq \max _{j, x} \sum_{x_{\alpha} \in A^{n}}\left|\sigma_{\alpha}^{\ell}\left(\xi_{\alpha}\right)\right| \delta_{x_{j} x} .
\end{aligned}
$$

$i$ is an arbitrary constituent of $\alpha$. The result does not depend on $i$ or $x$ because of translation invariance. We introduce

$$
\tilde{v}^{r}(0)=\sum_{x \in \mathbf{Z}^{3}}\left|v^{r}(x y)\right| .
$$

By translation invariance this is independent of $y$. If $v^{r}(x y) \geqq 0$, then $\tilde{v}^{r}$ is the Fourier transform of $v^{r}$.

We consider individual trees $\eta$ and numberings of the $\ell$-vertices (they are permuted by $\mathbf{S}$ ). We carry out the summations over variables $x_{\alpha}$ in expression (4.3) one by one, "trimming the tree and straightening its branches" as explained in Fig. 3. 


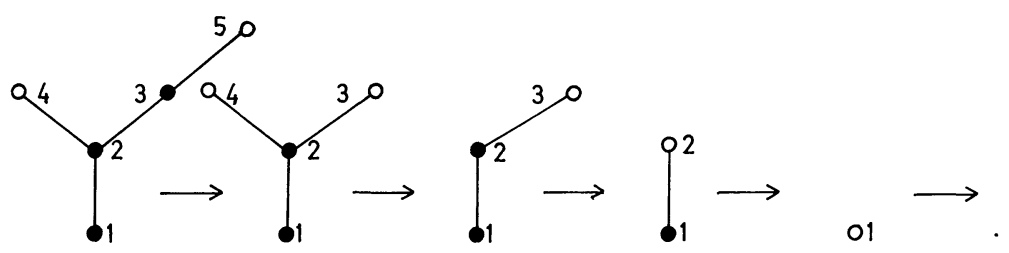

Fig. 3. How to trim a tree and straighten its branches. (The last dot represents a single constituent of the $\ell$-vertex $\alpha_{1}$ )

If we carry out the summations over $x_{\alpha_{a}}$ in the order $a=t, t-1, \ldots$, we are sure that in each step the summation variable is attached to a maximal vertex of the remaining tree, compare Fig. 2. Each $x_{\alpha}$-summation except the last is over all $x_{i} \in \mathbf{Z}^{3}$ for $i \underline{\underline{a}} \alpha$. In the last step, the position of one last constituent is exempt from summation. To do the individual $x_{\alpha}$-summations, we consider the terms in the sum over $j \underline{\epsilon} \alpha$ in expression (4.4) individually. The summation over $x_{j}$ is done last, with the help of (4.6). The preceding ones are carried out with the help of (4.5a). The freedom of selecting a constituent $i$ in (4.5a) is used to set $i=j$.

In this way all the $x$-summations can be done in sequence with the result that

$$
\begin{aligned}
\left\|\hat{\sigma}_{\alpha^{\prime}}^{\ell+1}\right\|\left(m_{\alpha^{\prime}}\right) \leqq & \frac{1}{t}\left[\beta \tilde{v}^{\ell}(0)\right]^{t-1} e^{-\beta \delta_{\ell}} \prod_{\alpha \in \alpha^{\prime}}\left\{\left\|\sigma_{\alpha}^{\ell}\right\|\left(m_{\alpha}\right) e^{-\beta \varepsilon_{\ell} \Sigma_{j \leqq \alpha} m_{j}^{2}}\right\} \\
& \cdot \mathbf{S} \int_{0}^{1} d s_{1} \ldots d s_{t-1} \sum_{\eta}\left\{\prod_{a=1}^{t-1} \sum_{j \in \alpha_{a}} \sum_{k \in \alpha_{\eta}(a)}\left|m_{j} m_{k}\right| s_{a-1} s_{a-2} \ldots s_{\eta(a)}\right\} .
\end{aligned}
$$

The sum over trees $\eta$ and integrations over $s$-variables can be performed with the help of the following.

Lemma 5 (The Tree Estimate). The following inequality holds for arbitrary $\mu(a) \geqq 0$ and $f$ as defined in Eq. (3.4)

$$
\sum_{\eta} \int_{0}^{1} d s_{1} \ldots d s_{t-1} f(\eta, \mathbf{s}) \prod_{a=1}^{t-1}[\mu(a+1) \mu(\eta(a))] \leqq \prod_{a=1}^{t-1}\left[\mu(a+1) e^{\mu(a)}\right] .
$$

The prototype of such an estimate was obtained by Glimm, Jaffe and Spencer in ref. 11. The reader who is not familiar with it can find a proof of inequality (4.8) in our Appendix C.

We set

$$
\mu(a)=\kappa_{\ell} \sum_{j \leqq \alpha_{a}}\left|m_{j}\right|, \quad\left(\kappa_{\ell} \geqq 0 \text { arbitrary }\right) .
$$

Applying inequality (4.8) with this choice of $\mu$ to expression (4.7) we obtain

$$
\begin{aligned}
\left\|\hat{\sigma}_{\alpha^{\prime}}^{\ell+1}\right\|\left(m_{\alpha^{\prime}}\right) \leqq & \frac{1}{t}\left[\beta \tilde{v}^{\ell}(0) / \kappa_{\ell}^{2}\right]^{t-1} e^{-\beta \delta_{\ell}} \prod_{\alpha \in \alpha^{\prime}} \\
& \cdot\left\{\left\|\sigma_{\alpha}^{\ell}\right\|\left(m_{\alpha}\right) \exp \sum_{j \leqq \alpha}\left(-\beta \varepsilon_{\ell} m_{j}^{2}+2 \kappa_{\ell}\left|m_{j}\right|\right)\right\} .
\end{aligned}
$$

We have used that $x \leqq e^{x}$ for $x \geqq 0$ to raise factors $\sum\left|m_{j}\right|$ into the exponent, and included some factors $\exp (\ldots)>1$ to make the resulting expression look more symmetrical. Inequality (4.10) is true for any choice of $\kappa_{\ell}>0$. 
Next we do $m$-summations. We introduce norms $[n=$ number of constituents of $\left.\alpha, \mathbf{Z}_{*}=\mathbf{Z}-(0)\right]$

$$
\left\|\sigma_{\alpha}^{\ell}\right\|_{\varepsilon, \kappa} \equiv \sum_{m_{\alpha} \in \mathbf{Z}_{\star}^{n}}\left\|\sigma_{\alpha}^{\ell}\right\|\left(m_{\alpha}\right) \exp \left[\sum_{j \leqq \alpha}\left(2 \kappa\left|\mathrm{m}_{j}\right|-\varepsilon \mathrm{m}_{j}^{2}\right)\right] .
$$

From definitions (4.5a), (4.11), (2.1) and inequality (4.5a) it follows that

$$
\int d \xi_{\alpha}\left|\sigma_{\alpha}^{\ell}\left(\xi_{\alpha}\right)\right| \leqq|\Lambda|\left\|\sigma_{\alpha}^{\ell}\right\|_{0,0}
$$

Inequality (4.10) implies that

$$
\left\|\hat{\sigma}_{\alpha^{\prime}}^{\ell+1}\right\|_{\varepsilon, \kappa} \leqq \frac{1}{t}\left[\beta \tilde{v}^{\ell}(0) / \kappa_{\ell}^{2}\right]^{t-1} e^{-\beta \dot{\delta}_{\ell}} \prod_{\alpha \in \alpha^{\prime}}\left\|\sigma_{\alpha}^{\ell}\right\|_{\varepsilon+\beta \varepsilon_{\ell}, \kappa+\kappa_{\ell}} .
$$

Finally we can estimate sums over types of vertices. Consider sums of $(\ell+1)$ vertices $\alpha^{\prime}$ which consist of a given number $\left|\alpha^{\prime}\right|=t$ of $\ell$-vertices. Define

$$
\begin{aligned}
\left\|\sigma_{t}^{\ell+1}\right\|_{\varepsilon, \kappa} & \equiv \sum_{\substack{\left[\alpha^{\prime}\right] \in T \in t+1 \\
\left|\alpha^{\prime}\right|=t}}\left\|\sigma_{\alpha^{\prime}}^{\ell+1}\right\|_{\varepsilon, \kappa} \\
& =\sum_{\substack{\left[\alpha^{\prime}\right] \in T_{\ell+1}\left|\alpha^{\prime}\right|=t}}\left(\frac{t !}{\prod_{[\beta] \in T_{\ell}} N_{[\beta]}^{\alpha^{\prime} !}}\right)\left\|\hat{\sigma}_{\alpha^{\prime}}^{\ell+1}\right\|_{\varepsilon, \kappa} .
\end{aligned}
$$

Ultimately we will be interested in estimating the left hand side of (2.27). Because of inequality (4.12) it satisfies the bound

$$
\frac{1}{|\Lambda|} \sum_{\left[\alpha^{\prime}\right] \in T_{R}} \int d \xi_{\alpha^{\prime}}\left|\sigma_{\alpha^{\prime}}^{R}\left(\xi_{\alpha^{\prime}}\right)\right| \leqq \sum_{t \geqq 1}\left\|\sigma_{t}^{R}\right\|_{0,0}
$$

It suffices therefore to estimate sums of the form $\sum_{t \geqq 1}\left\|\sigma_{t}^{\ell}\right\|_{\varepsilon, \kappa}$. We derive a recursive bound for them.

We insert the estimates (4.13) on the right hand side of (4.14). This gives

$$
\begin{aligned}
\left\|\sigma_{t}^{\ell+1}\right\|_{\varepsilon, \kappa} \leqq & \frac{1}{t}\left[\beta \tilde{v}^{\ell}(0) / \kappa_{\ell}^{2}\right]^{t-1} e^{-\beta \delta_{\ell}} \sum_{\substack{\left[\alpha^{\prime}\right] \in T_{\ell}\left|\alpha^{\prime}\right|=t+1 \\
\mid \alpha^{\prime}}} \cdot\left(\frac{t !}{\prod_{[\beta] \in T_{\ell}} N_{[\beta]}^{\alpha^{\prime} !}}\right) \prod_{\alpha \in \alpha^{\prime}}\left\|\sigma_{\alpha}^{\ell}\right\|_{\varepsilon+\beta \varepsilon_{\ell}, \kappa+\kappa_{\ell}} .
\end{aligned}
$$

The type $\left[\alpha^{\prime}\right]$ of a $(\ell+1)$-vertex is given by the number of times $N_{[\beta]}^{\alpha^{\prime}}$ with which any given type $[\beta]$ of $\ell$-vertex appears in it. Summation over $\left[\alpha^{\prime}\right]$ is therefore equivalent to summation over the multiplicities $N_{[\beta]}^{\alpha^{\prime}}$ subject to the constraint that the total number of $\ell$-vertices $\sum_{[\beta]} N_{[\beta]}^{\alpha^{\prime}}=t$. We can therefore apply the multinomial theorem to do the $\left[\alpha^{\prime}\right]$-summations. As a result

$$
\begin{aligned}
\left\|\sigma_{t}^{\ell+1}\right\|_{\varepsilon, \kappa} \leqq & \frac{1}{t}\left[\beta \tilde{v}^{\ell}(0) / \kappa_{\ell}^{2}\right]^{t-1} \\
& \cdot\left(\sum_{[\alpha] \in T_{\ell}}\left\|\sigma_{\alpha}^{\ell}\right\|_{\varepsilon+\beta \varepsilon_{\ell}, \kappa+\kappa_{\ell}}\right)^{t} e^{-\beta \delta_{\ell}} .
\end{aligned}
$$


We insert definition (4.14) to rewrite this as

$$
\begin{aligned}
\sigma_{t}^{\ell+1} \|_{\varepsilon, \kappa} \leqq & \frac{1}{t}\left[\beta \tilde{\nu}^{\ell}(0) / \kappa_{\ell}^{2}\right]^{t-1} \\
& \cdot\left(\sum_{u=1,2, \ldots}\left\|\sigma_{u}^{\ell}\right\|_{\varepsilon+\beta \varepsilon_{\ell}, \kappa+\kappa_{\ell}}\right)^{t} e^{-\beta \delta_{\ell}} .
\end{aligned}
$$

This is valid for $\ell \geqq 1$ and for any $\kappa_{\ell}>0$. Finally we may sum over $t$. This produces our

Proposition 6. If the potential of a lattice gas with pair interactions can be split into $R$ pieces $v^{\ell}(\ell=0 \ldots R-1)$, which are of the form (2.6), (4.1) and satisfy the bounds (4.2), then the following recursive bounds on sums of vertex functions are valid for $\ell \geqq 1$ and arbitrary $\kappa_{\ell}>0$ :

$$
\begin{aligned}
\sum_{t=1,2, \ldots}\left\|\sigma_{t}^{\ell+1}\right\|_{\varepsilon, \kappa} \leqq & -\left[\beta \tilde{v}^{\ell}(0) / \kappa_{\ell}^{2}\right]^{-1} e^{-\beta \delta_{\ell}} \\
& \cdot \ln \left(1-\beta \tilde{v}^{\ell}(0) \kappa_{\ell}^{-2} \sum_{u=1,2, \ldots}\left\|\sigma_{u}^{\ell}\right\|_{\varepsilon+\beta \varepsilon_{\ell}, \kappa+\kappa_{\ell}}\right),
\end{aligned}
$$

provided the argument of the logarithm is positive.

To apply these bounds, one starts from a bound on $\sigma^{1}$ and applies the recursive bound (4.17) repeatedly. This produces for instance

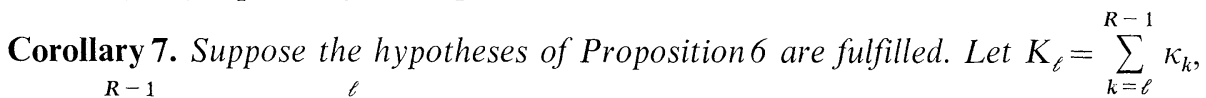
$E_{\ell}=\beta \sum_{k=\ell}^{R-1} \varepsilon_{k}$ and $\Delta_{\ell}=\sum_{k=1}^{\ell} \delta_{k}$. Suppose that the following inequalities are also fulfilled for some $A>0,0<C<1$.

$$
\begin{gathered}
\sum_{n=1,2, \ldots}\left\|\sigma_{n}^{1}\right\|_{E_{1}, K_{1}} \leqq A(1-C)^{-1} \\
\beta \tilde{v}^{\ell}(0) \kappa_{\ell}^{-2} \leqq C(1-C)^{\ell} A^{-1} e^{\beta A_{\ell-1}} \quad \text { for } \quad \ell=1 \ldots R-1 .
\end{gathered}
$$

Then

$$
|\Lambda|^{-1} \sum_{[\alpha] \in T_{R}} \int d \xi_{\alpha}\left|\sigma_{\alpha}^{R}\left(\xi_{\alpha}\right)\right| \leqq A(1-C)^{-R} e^{-\beta \Delta_{R-1}} .
$$

Proof. We show that

$$
\sum_{n=1,2, \ldots}\left\|\sigma_{n}^{\ell}\right\|_{E_{\ell}, K_{\ell}} \leqq A(1-C)^{-\ell} e^{-\beta \Delta_{\ell-1}} \quad \text { for } \quad \ell=1 \ldots R .
$$

The assertion of Corollary 7 follows from the special case $\ell=R$ of this by inequality (4.15). The proof of (4.20) proceeds by induction. If $\ell=1$ inequality (4.20) is true by hypothesis (4.18a). Suppose (4.20) is true for some $\ell \geqq 1$; we show it is also true for $\ell+1$. Since $-\ln (1-x) \leqq x(1-x)^{-1}$ for $1>x \geqq 0$, the recursive bound (4.17) gives

$$
\begin{aligned}
\sum_{n=1,2, \ldots}\left\|\sigma_{n}^{\ell+1}\right\|_{E_{\ell+1}, K_{\ell+1}} \leqq & A(1-C)^{-\ell} e^{-\beta \Delta_{\ell-1}} e^{-\beta \delta_{\ell}} \\
& \cdot\left(1-\beta \tilde{\nu}^{\ell}(0) \kappa_{\ell}^{-2} A(1-C)^{-\ell} e^{-\beta \Delta_{\ell-1}}\right)^{-1} \\
\leqq & A(1-C)^{-\ell-1} e^{-\beta \Delta_{\ell}} .
\end{aligned}
$$

The second inequality follows from hypothesis (4.18b). Proof completed. 


\section{Application to Yukawa Gases}

We will now apply the results obtained so far to the Yukawa gas models that were described at the end of the introduction. We split the potential as in Eq. (2.5) into the hard core potential $v^{0}$ plus $R-1=2$ pieces $v^{1}$ and $v^{2}$. Because of the presence of the hard core, an arbitrary finite value can be assigned to $v^{1}\left(\xi_{1}, \xi_{2}\right)$ for $x_{1}=x_{2}$. We make use of this freedom to set

$$
\begin{gathered}
v^{1}\left(\xi_{1}, \xi_{2}\right)= \begin{cases}0 & \text { if } x_{1}=x_{2}, \operatorname{sign} m_{1}=-\operatorname{sign} m_{2} \\
m_{1} m_{2} v^{1}\left(x_{1} x_{2}\right) & \text { otherwise, }\end{cases} \\
v^{1}\left(x_{1} x_{2}\right)=\left(-\Delta+M_{1}^{2}\right)^{-1}\left(x_{1}, x_{2}\right),
\end{gathered}
$$

and

$$
\begin{gathered}
v^{2}\left(\xi_{1}, \xi_{2}\right)=m_{1} m_{2} v^{2}\left(x_{1} x_{2}\right), \\
v^{2}\left(x_{1} x_{2}\right)=\left(-\Delta+M^{2}\right)^{-1}\left(x_{1}, x_{2}\right)-\left(-\Delta+M_{1}^{2}\right)^{-1}\left(x_{1}, x_{2}\right) .
\end{gathered}
$$

$M_{1} \geqq M$ will be chosen later on. First we will now establish

Proposition 8. This choice of $v^{1}, v^{2}$ satisfies the bounds (4.2) for some $\varepsilon_{1}>0$ if $M_{1}$ is sufficiently small, with $\varepsilon_{2}=\delta_{2}=0$,

$$
\gamma_{1}=\frac{1}{2} v^{1}(0) \equiv \frac{1}{2} v^{1}(x x), \quad \text { and } \quad 0<\varepsilon_{1}<\frac{1}{2} v^{1}(0) \text {. }
$$

Moreover, $v^{\ell}\left(x_{1} x_{2}\right) \geqq 0$ and

$$
\tilde{v}^{1}(0)=M_{1}^{-2}, \quad \tilde{v}^{2}(0)=M^{-2}-M_{1}^{-2}<M^{-2} .
$$

Proof. The positivity assertion is a well-known fact (see Appendix D). It follows that $\tilde{v}^{1}(0)$ as defined by Eq. (4.5) are given by the Fourier transforms

$$
\tilde{v}^{\ell}(k)=\sum_{x \in \mathbf{Z}^{3}} e^{i k x} v^{\ell}(x 0)
$$

Explicitly

$$
\begin{gathered}
\tilde{v}^{1}(k)=\left(2 \sum_{\mu=1}^{3}\left[1-\cos k_{\mu}\right]+M_{1}^{2}\right)^{-1}, \\
\tilde{v}^{2}(k)=\left(2 \sum_{\mu=1}^{3}\left[1-\cos k_{\mu}\right]+M^{2}\right)^{-1}-\left(2 \sum_{\mu=1}^{3}\left[1-\cos k_{\mu}\right]+M_{1}^{2}\right)^{-1} .
\end{gathered}
$$

Relations (5.3) follow by setting $k=0$.

The self energy $\frac{1}{2} v^{1}(0)$ of a particle of unit charge is given by

$$
v^{1}(0)=(2 \pi)^{-3} \int_{\left|k_{\mu}\right| \leqq \pi} d^{3} k\left(2 \sum_{\mu}\left[1-\cos k_{\mu}\right]+M_{1}^{2}\right)^{-1},
$$

which we compare with the Coulomb potential $v_{C b}(x-y)=(-\Delta)^{-1}(x y)$. A short calculation shows that

$$
v^{1}(0)=v_{C b}(0)-(4 \pi)^{-1} M_{1}+O\left(M_{1}^{2}\right) .
$$


The numerical value of $v_{C b}(0)$ is known [12]

$$
v_{C b}(0)=0.252731 \text {. }
$$

Now we are ready to derive the bounds (4.2). We introduce the Hilbert space $H$ of complex functions $f$ on $\mathbf{Z}^{3}$ with scalar product

$$
(f, g)=\sum_{x \in \mathbf{Z}^{3}} \bar{f}(x) g(x) .
$$

The kernels $\nu^{\ell}(x y)$ specify positive operators in $H$ which act according to

$$
\left(v^{\ell} f\right)(x)=\sum_{y \in \mathbf{Z}^{3}} v^{\ell}(x y) f(y)
$$

These operators are diagonalized by Fourier transform, and it follows from Eqs. (5.5) that

$$
\left(m, v^{\ell} m\right) \geqq \varrho^{\ell}(m, m),
$$

with

$$
\varrho^{1}=\left(12+M_{1}^{2}\right)^{-1} ; \quad \varrho^{2}=0
$$

Consider a set of $N$ particles with integer charges $m_{i} \neq 0$ and not necessarily distinct positions $x_{i}$. Set

$$
m(x)=\sum_{i} m_{i} \delta_{x_{\imath} x}
$$

It follows from inequality (5.8) that $v^{2}$ satisfies a bound (4.2) with

$$
\delta_{2}=\varepsilon_{2}=0 \text {. }
$$

It remains to study $v^{1}$. From definition (5.1a) it follows that

$$
E \equiv \frac{1}{2} \sum_{i, j} v^{1}\left(\xi_{i}, \xi_{j}\right)=\frac{1}{2}\left(m, v^{1} m\right)+\frac{1}{2} \sum_{\substack{i, j \\ x_{i}=x_{j}, \operatorname{sign} m_{\mathrm{r}}=-\operatorname{sign} m_{J}}}\left|m_{i} m_{j}\right| v^{1}(0) .
$$

Inserting the bound (5.8) for the first term and noting that $\varrho^{1} \leqq v^{1}(0)$, one finds after a short calculation that

$$
\frac{1}{2} \sum_{i, j} v^{1}\left(\xi_{i}, \xi_{j}\right) \geqq \frac{1}{2} \varrho^{1} \sum m_{i}^{2} .
$$

If $M_{1}$ is sufficiently small one has $4 Q^{1}>v^{1}(0)$ by Eqs. (5.6), (5.7). Therefore inequality (5.11) implies validity of bounds of the form (4.2) with $\gamma_{1}=\frac{1}{2} v^{1}(0)$ if $M_{1}$ is sufficiently small and $\sum m_{i}^{2} \geqq 4$.

It remains to discuss the case $\sum m_{i}^{2} \leqq 3$. There are only the following possibilities. One may have $N=1,2$ or 3 particles with charges $m_{i}= \pm 1$. Consider first particles whose positions are all distinct, and define for this case

$$
E_{N}=\min _{\left\{x_{i}, m_{\imath}= \pm 1\right\}} \frac{1}{2} \sum_{i, j=1}^{N} m_{i} m_{j} v^{1}\left(x_{i} x_{j}\right)
$$


$v^{1}(x y)$ is positive and monotonically decreasing in the modulus $\left|x_{\mu}-y_{\mu}\right|$ of each coordinate difference (see Appendix D). It follows that

$$
\begin{aligned}
& E_{1}=\frac{1}{2} v^{1}(0), \\
& E_{2}=v^{1}(0)-v^{1}(1)=\frac{1}{6}\left[1-M_{1}^{2} v^{1}(0)\right], \\
& E_{3} \geqq \frac{3}{2} v^{1}(0)-2 v^{1}(1) .
\end{aligned}
$$

(We use the abbreviation $v^{1}(1)=v^{1}\left(x, x+e_{\mu}\right), e_{\mu}=$ unit vector in the $\mu$-direction.) $E_{2}$ is the energy of a dipole $=$ two charges \pm 1 at nearest neighbour sites of the lattice. It can be computed because

$$
v^{1}(0)-v^{1}(1)=-\frac{1}{6} \sum_{ \pm, \mu}\left[v^{1}\left( \pm e_{\mu} 0\right)-v^{1}(00)\right]=-\frac{1}{6} \Delta v^{1}(0) .
$$

The inequality for $E_{3}$ is obtained by dropping the repulsive interaction between like charges. In the limit $M_{1}=0$, relations (5.13) give $E_{1}=\frac{1}{2} v_{C b}(0)=0.126_{4}, E_{2}$ $=0.166_{6}, E_{3} \geqq 0.206$. Thus $E_{N}>\frac{1}{2} v^{1}(0)$ for $N=2,3$ if $M_{1}$ is sufficiently small. So the desired bound (4.2) holds.

Finally we have to dispose of the possibility that (at least) two of the two or three charges sit on the same lattice site. If they have equal charges, the argument is the same as above. It they have opposite charges, they do not interact with a remaining third particle, and the attractive potential between them has to be ignored according to Eq. (5.1). It follows that $E \geqq \frac{1}{2} N v^{1}(0)$ and the desired bound (4.2) is again satisfied. This completes the proof of Proposition 8.

To apply the recursive bounds on vertex functions of Sect. 4 we need a bound in $\sigma^{1}$ first. It is provided by

Lemma 9. $\sum_{n=1,2, \ldots}\left\|\sigma_{n}^{1}\right\|_{\varepsilon, \kappa} \leqq 2|\lambda(1)| e^{2 \kappa-\varepsilon}(1-C)^{-1}$ if $1+\bar{z}(1+2|\zeta|) \leqq C e^{\varepsilon-2 \kappa}$. This is true for any $C$ in the interval $0<C<1$ and $\bar{z} \geqq 0$.

Proof. There is only one type of 1-vertex with $n$ constituents. It follows therefore from the explicit formula (3.2) for $\sigma^{1}$ that

$$
\left\|\sigma_{n}^{1}\right\|_{\varepsilon, \kappa}=\frac{1}{n}\left[\sum_{q= \pm 1, \pm 2, \ldots}|\lambda(q)| \exp \left(-\varepsilon q^{2}+2 \kappa|q|\right)\right]^{n} .
$$

The modified Bessel functions satisfy $I_{n+1}(2 \bar{z}) \leqq \bar{z} I_{n}(2 \bar{z})$ if $\bar{z} \geqq 0, n=0,1, \ldots$. This follows from their series representation [4]. Therefore

$$
\begin{aligned}
|\lambda(q)| & \leqq|\lambda(1)| \bar{z}^{|q|-1} \quad(q= \pm 1, \pm 2, \ldots) ; \\
\lambda(1) & \equiv \zeta^{\prime} \bar{z} \quad \text { with } \quad\left|\zeta^{\prime}\right| \leqq|\zeta| .
\end{aligned}
$$

for all three models (1.14). (We adopt the convention (1.15).) We insert this into (5.14). We must estimate

$$
S=\sum_{q= \pm 1, \pm 2, \ldots}\left(\bar{z} e^{2 \kappa}\right)^{|q|} e^{-\varepsilon q^{2}} .
$$

We distinguish two cases $\bar{z} e^{2 \kappa} \gtrless 1$. Consider first the case $\bar{z} e^{2 \kappa} \geqq 1$. In this case we can estimate

$$
S \leqq \sum_{q}\left(\bar{z} e^{2 \kappa} e^{-\varepsilon}\right)^{q^{2}} \leqq 2 \sum_{n=1,2, \ldots}\left(\bar{z} e^{2 \kappa-\varepsilon}\right)^{n}=2 A(1-A)^{-1}
$$


with $A=\bar{z} e^{2 \kappa-\varepsilon}$. Thus

$$
\begin{aligned}
\sum_{n \geqq 1}\left\|\sigma_{n}^{1}\right\|_{\varepsilon, \kappa} & \leqq \sum_{n \geqq 1} \frac{1}{n}\left[2\left|\zeta^{\prime}\right| A(1-A)^{-1}\right]^{n}=-\ln \left[1-2\left|\zeta^{\prime}\right| A(1-A)^{-1}\right] \\
& \leqq 2\left|\zeta^{\prime}\right| A\left[1-\left(1+2\left|\zeta^{\prime}\right|\right) A\right]^{-1}, \quad \text { if } \quad 2|\zeta| A(1-A)^{-1}<1
\end{aligned}
$$

[Here and in the following the inequality $-\ln (1-x) \leqq x(1-x)^{-1}$ for $1>x \geqq 0$ is used whenever a logarithm appears.] The inequality of Lemma 9 will therefore hold if $\left(1+2\left|\zeta^{\prime}\right|\right) A \equiv\left(1+2\left|\zeta^{\prime}\right|\right) \bar{z} e^{2 \kappa-\varepsilon} \leqq C<1$. The hypothesis of Lemma 9 guarantees that this is satisfied since $\left|\zeta^{\prime}\right| \leqq|\zeta|$ by $(5.15)$. Therefore Lemma 9 is proven for $\bar{z} e^{2 \kappa} \geqq 1$. In the second case, $\bar{z} e^{2 \kappa} \leqq 1$, we estimate

$$
S \leqq \bar{z} e^{2 \kappa} \sum_{q} e^{-\varepsilon q^{2}} \leqq 2 \bar{z} e^{2 \kappa} \sum_{n \geqq 1} e^{-\varepsilon n}=2 \bar{z} e^{2 \kappa-\varepsilon}\left(1-e^{-\varepsilon}\right)^{-1} .
$$

Then one proceeds as before. The details are left to the reader. One finds that the assertion of Lemma 9 also holds in this case. This completes the proof of Lemma 9.

Proof of Theorem 1. Because of Proposition 3, it suffices to show that the hypotheses of Corollary 7 can be fulfilled by a suitable choice of $M_{1} \geqq M$ if the hypotheses of Theorem 1 are fulfilled. Corollary 7 is valid for any choice of $\kappa_{\ell}>0$; $\varepsilon_{\ell}$ and $\delta_{\ell}$ are given by Proposition 8 , and $R=3$. The hypotheses of Proposition 6 are met by Proposition 8 . We set

$$
M_{1}=\max \left(M, 4 \pi \kappa_{1} \beta^{-1}\right), \quad \kappa_{1}=\kappa_{2}=-\ln (1-C) .
$$

The following considerations are valid for any $C$ in the interval $0<C<1$. In the end we may set $C=\frac{1}{3}$.

By Lemma 9

$$
\sum_{n \geqq 1}\left\|\sigma_{n}^{1}\right\|_{\beta \varepsilon_{1}, \kappa_{1}+\kappa_{2}} \leqq A(1-C)^{-1},
$$

with $\left(\zeta^{\prime} \equiv \lambda(1) / \bar{z}\right.$ as in $\left.(5.15)\right)$

provided

$$
A=2\left|\zeta^{\prime}\right| e^{-\beta \varepsilon_{1}+2\left(\kappa_{1}+\kappa_{2}\right)}=2\left|\zeta^{\prime}\right| \bar{z} e^{-\beta \varepsilon_{1}}(1-C)^{-4},
$$

$$
1+\bar{z}(1+2|\zeta|) \leqq C(1-C)^{4} e^{\beta \varepsilon_{1}}
$$

This is assured if hypothesis (1.17b) of Theorem 1 is fulfilled, and $\beta$ is sufficiently large or $C_{2}$ sufficiently small, depending on $C$. Thus, hypothesis (4.18a) of Corollary 7 is fulfilled.

Next we turn to hypothesis $(4.18 \mathrm{~b})$ for $\ell=1$. By Proposition $8, \tilde{v}^{1}(0)=M_{1}^{-2}$. Therefore it is required that

$$
\beta M_{1}^{-2} \kappa_{1}^{-2} \leqq C(1-C)^{5}\left(2\left|\zeta^{\prime}\right| \bar{z}\right)^{-1} e^{\beta \varepsilon_{1}} .
$$

Since $M_{1}^{-2} \leqq(4 \pi)^{-2} \kappa_{1}^{-2} \beta^{2}$ and $\kappa_{1} \geqq C$ this is fulfilled if

$$
2\left|\zeta^{\prime}\right| \bar{z} \leqq(4 \pi)^{2} C^{3}(1-C)^{5} e^{\beta \varepsilon_{1}} \beta^{-3} .
$$

This is assured by hypothesis (1.17b) of Theorem 1 if $C_{2}$ is sufficiently small, depending on $C$, since $\left|\zeta^{\prime}\right| \leqq|\zeta|$ by $(5.15)$. 
Finally we have to verify hypothesis $(4.18 \mathrm{~b})$ for $\ell=2$. Since $\tilde{v}^{2}(0)<M^{-2}$ it will be satisfied if

$$
\beta M^{-2} \kappa_{2}^{-2} \leqq C(1-C)^{6}\left(2\left|\zeta^{\prime}\right| \bar{z}\right)^{-1} e^{\beta \gamma_{1}}
$$

where $\gamma_{1}=\varepsilon_{1}+\delta_{1}=\frac{1}{2} v^{1}(0)$ by Proposition 8 . Since $\kappa_{2} \geqq C$ this holds if

$$
M^{2} \geqq C^{-3}(1-C)^{-6} 2 \beta\left|\zeta^{\prime}\right| \bar{z} e^{-\beta v^{1}(0) / 2} .
$$

This is of the form of hypothesis (1.17a) of Theorem 1 , except that $v^{1}(0)$ appears in place of $v_{C b}(0)$. We distinguish the two cases $M_{1}=M$ and $M_{1}=4 \pi \kappa_{1} \beta^{-1}$. We have $M_{1}=M$ only if $M \geqq 4 \pi \kappa_{1} \beta^{-1}$. In this case, (5.23) follows from (1.17b) if $C_{2}$ is sufficiently small. If $M_{1}=4 \pi \kappa_{1} \beta^{-1}$ we can use Eq. (5.6) to obtain

$$
\beta v^{1}(0)=\beta v_{C b}(0)-\kappa_{1}+\beta O\left(\beta^{-2}\right) \geqq \beta v_{C b}(0)+2 \ln (1-C)
$$

if $\beta$ is sufficiently large. Therefore (5.23) holds if $\beta$ is sufficiently large and

$$
M^{2} \geqq C^{-3}(1-C)^{-7} 2 \beta\left|\zeta^{\prime}\right| \bar{z} e^{-\beta v_{C b}(0) / 2} .
$$

This is true if hypothesis (1.17a) of Theorem 1 holds, with $C_{1}$ sufficiently small (depending on $C$ ), since $\zeta^{\prime} \bar{z}=\lambda(1)$ by definition (5.15). Validity of all the hypotheses of Corollary 7 has now been established, and the proof of Theorem 1 is therefore complete.

Remark. Corollary 7 also tells us that

$$
\begin{aligned}
\sum_{n \geqq 1}\left|b_{n}\right||\zeta|^{n} & \leqq|\Lambda|^{-1} \sum_{[\alpha] \in T_{3}} \int d \xi_{\alpha}\left|\sigma_{\alpha}^{3}\left(\xi_{\alpha}\right)\right| \\
& \leqq 2|\lambda(1)| e^{-\beta v^{1}(0) / 2}(1-C)^{-7}
\end{aligned}
$$

The leading term in the sum comes from a 3-vertex with only a single constituent. Its contribution is equal to

$$
b_{1} \zeta=\sum_{q= \pm 1 . \pm 2, \ldots} \lambda(q) e^{-\beta v(0) q^{2} / 2} \sim 2 \lambda(1) e^{-\beta v(0) / 2} \quad \text { for large } \beta
$$

Thus, inequality (5.25) estimates the whole sum by a multiple of the first term. $v^{1}$ is obtained from $v$ by substituting $M_{1}$ for $M$. Noting that $v^{1}=v$ if $M_{1}=M$, we deduce from Eqs. (5.6), (5.16) that $v(0)-v^{1}(0) \leqq \beta^{-1} \ln (1-C)^{-1}+O\left(\beta^{-2}\right)$. The constant $C$ can be made arbitrarily small if the constants $C_{1}$ and $C_{2}$ in Theorem 1 are chosen sufficiently small, depending on $C$.

\section{Appendix A. Proof of Exponentiation of the Grand Partition Function, (Proposition 3)}

We want to prove formula (2.30) starting from Eq. (2.29). We consider $Z_{\Lambda}^{N}$ as a function of the quantities

$$
A([\gamma])=\int d \xi_{\gamma} \sigma_{\gamma}^{R}\left(\xi_{\gamma}\right), \quad Z_{\Lambda}^{N}=Z_{\Lambda}^{N}(\{A([\gamma])\})
$$


Under the transformation $A([\gamma]) \rightarrow \lambda^{|C(\gamma)|} A([\gamma]), Z_{\Lambda}^{N}$ scales in the following way, as can be seen from (2.29) by simple inspection:

$$
Z_{\Lambda}^{N}\left(\left\{\lambda^{|C(\gamma)|} A([\gamma])\right\}\right)=\lambda^{N} Z_{\Lambda}^{N}(\{A([\gamma])\}) .
$$

Differentiating (A.1) with respect to $\lambda$ and setting $\lambda=1$ afterwards, we obtain the identity:

$$
N Z_{\Lambda}^{N}=\sum_{[\gamma] \in T_{R}}|C(\gamma)| A([\gamma]) \frac{\partial Z_{\Lambda}^{N}}{\partial A([\gamma])} .
$$

For further investigation we have to compute $\frac{\partial Z_{\Lambda}^{N}}{\partial A([\gamma])}$ :

$$
\begin{aligned}
& \frac{\partial Z_{A}^{N}}{\partial A([\gamma])}=\frac{\partial}{\partial A([\gamma])} \sum_{\substack{\left\{N_{[\beta]}^{R}\right\}\{, \beta] \in I_{\alpha} \\
\sum \beta N_{[\beta]}^{R}|C(\beta)|=N}} \prod_{[\alpha] \in T_{R}} \frac{A([\alpha])^{N_{[\alpha]}^{R}}}{N_{[\alpha]}^{R} !}
\end{aligned}
$$

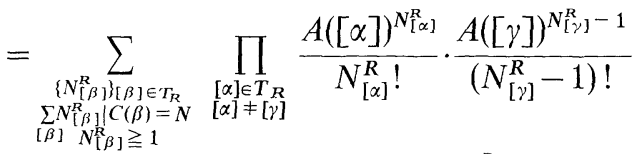

$$
\begin{aligned}
& =\sum_{\substack{\sum_{[\beta]}^{R} N_{[\beta]}^{R}\left\{N_{[\beta]\}}^{R}[\beta) \mid=N\right] \in T_{R} \\
[\beta-|C(\gamma)|}} \prod_{[\alpha] \in T_{R}} \frac{A([\alpha])^{N_{[\alpha]}^{R}}}{N_{[\alpha]}^{R} !}=Z_{\Lambda}^{N-|C(\gamma)|},
\end{aligned}
$$

if $|C(\gamma)| \leqq N$, otherwise $\frac{\partial Z_{\Lambda}^{N}}{\partial A([\gamma])}=0$.

So we get the following equation for the quantities $Z_{A}^{N}$ :

$$
Z_{\Lambda}^{N}=\sum_{\substack{[\gamma] \in T_{R} \\|C(\gamma)| \leqq N}} \frac{|C(\gamma)|}{N} A([\gamma]) Z_{\Lambda}^{N-|C(\gamma)|} .
$$

From (A.4) we can derive (2.30) by a simple computation; we introduce a fugacity $z$ and consider $z \frac{\partial}{\partial z} Z_{A}$ :

$$
\begin{aligned}
z \frac{\partial}{\partial z} \sum_{N} z^{N} Z_{\Lambda}^{N} & =\sum_{N} N z^{N} Z_{\Lambda}^{N} \\
& =\sum_{N} \sum_{\substack{[\gamma] \in T_{R} \\
\mid C(\gamma) ! N}}|C(\gamma)| A([\gamma]) z^{|C(\gamma)|} Z_{\Lambda}^{N-|C(\gamma)|} z^{N-|C(\gamma)|} .
\end{aligned}
$$

The left hand side is an entire function of $z$ (a polynomial in our case because of the hard core). Assuming absolute convergence of $\sum_{[\gamma] \in T_{R}} z^{|C(\gamma)|} A([\gamma])$ for $|z| \leqq 1$, we conclude: (changing the summation over $N$ and $[\gamma]$ )

$$
\begin{aligned}
z \frac{\partial}{\partial z} \sum_{N} z^{N} Z_{\Lambda}^{N}= & \left\{\sum_{[\gamma] \in T_{R}}|C(\gamma)| z^{|C(\gamma)|} A([\gamma])\right\} \\
& \cdot \sum_{N} z^{N} Z_{\Lambda}^{N} .
\end{aligned}
$$


Eq. (A.6) implies

$$
\sum_{N} z^{N} Z_{A}^{N}=\exp \sum_{[\gamma] \in T_{R}} z^{|C(\gamma)|} A([\gamma])
$$

for $|z| \leqq 1$. This proves Proposition 3 .

\section{Appendix B. Proof of the Tree Formula (Proposition 4)}

Suppose we have a set of indices $I$ and an array of real numbers $w(i j)=w(j i)$ for $i, j \in I$. We assume that some ordering on $I$ is given. For any finite subset $\alpha \subset I$ we define the following quantities:

$$
\begin{aligned}
& Q(\alpha)=\exp \sum_{1 \leqq j<k \leqq n} w\left(\alpha_{j} \alpha_{k}\right), \quad \text { if } \quad \alpha=\left\{\alpha_{1}, \ldots, \alpha_{n}\right\}, \quad \alpha_{1}<\alpha_{2}<\ldots<\alpha_{n} \\
& Q(\emptyset)=1 \text {, } \\
& f_{\alpha_{j} \alpha_{k}}=e^{w\left(\alpha_{j} \alpha_{k}\right)}-1 \text {, } \\
& A(\alpha)=\frac{1}{n !} \sum_{\mathscr{G}_{\alpha}} \prod_{\left(\alpha_{j} \alpha_{k}\right) \in \mathscr{G}_{\alpha}} f_{\alpha_{j} \alpha_{k}}, \quad \text { if } \quad \alpha=\left\{\alpha_{1}, \ldots, \alpha_{n}\right\} \\
& A(\emptyset)=0, \quad A(\alpha)=1 \quad \text { for } n=1 .
\end{aligned}
$$

Summation over $\mathscr{G}_{\alpha}$ is over all connected graphs, i.e. sets of pairs $\left(\alpha_{j}, \alpha_{k}\right)$ of elements in $\alpha$, where each pair $\left(\alpha_{j}, \alpha_{k}\right)$ occurs at most once in $\mathscr{G}_{\alpha}$. We want to show the following proposition:

\section{Proposition B.1.}

$$
A(\alpha)=\frac{1}{n} \mathbf{S}_{\alpha} \int_{0}^{1} d s_{1} \ldots d s_{n-1} \prod_{k=2}^{n} \partial_{s_{k-1}} W\left(s_{1} \ldots s_{k-1} \mid 1 \ldots k\right) e^{W\left(s_{1} \ldots s_{k-1} \mid 1 \ldots n\right)},
$$

where $S_{\alpha}$ denotes the symmetrization with respect to the variables $\alpha_{1} \ldots \alpha_{n}$ and

$$
\begin{gathered}
W\left(s_{1} \ldots s_{m-1} \mid 1 \ldots m\right)=\sum_{1 \leqq j<k \leqq m} s_{j} \ldots s_{k-1} w\left(\alpha_{j} \alpha_{k}\right) \quad[m=2,3, \ldots, n], \\
W(\mid 1)=0 \quad[m=1] .
\end{gathered}
$$

Remarks (i) We expand the product in (B.4) and obtain, using the notations and definitions of Sect. 3:

$$
\prod_{k=2}^{n} \partial_{s_{k-1}} W\left(s_{1} \ldots s_{k-1} \mid 1 \ldots k\right)=\sum_{\eta} f(\eta, \mathbf{s}) \prod_{k=1}^{n-1} w\left(\alpha_{k+1} \alpha_{\eta(k)}\right) .
$$

Summation over $\eta$ is over all trees on $n$ vertices and $\mathbf{s}=\left(s_{1}, \ldots, s_{n-1}\right)$. From (B.6) we see that Eq. (B.4) is in some sense a generalized tree formula.

(ii) Proposition 4 in Sect. 3 follows from Proposition B.1 by setting: $I=\left\{\alpha_{1}, \alpha_{2}, \ldots, \alpha_{t}\right\}$ [where $\alpha_{1}, \ldots, \alpha_{t}$ label the subvertices of the $(\ell+1)$-vertex $\left.\alpha^{\prime}\right]$ and $w\left(\alpha_{j} \alpha_{k}\right)=-\beta v^{\ell}\left(\alpha_{j} \alpha_{k}\right)$

$$
-\beta W\left(s \mid \alpha^{\prime}\right)=W\left(s_{1} \ldots s_{t-1} \mid 1 \ldots t\right)-\beta \sum_{j} v^{\ell}\left(\alpha_{j} \alpha_{j}\right) \text { for } \quad \alpha^{\prime}=\left\{\alpha_{1}, \ldots, \alpha_{t}\right\} .
$$

To see this compare (B.3) with (2.11) and (B.4), (B.6) with (3.7). 
To prove Proposition B.1 we need the following two lemmata. They are in some sense two Kirkwood-Salsburg equations: $Q\left(\alpha^{\prime}\right)$ corresponds to Boltzmann factors and $A\left(\alpha^{\prime}\right)$ to Ursell functions.

\section{Lemma B.2.}

$$
Q(\alpha)=\frac{1}{n} \sum_{S \subset \alpha}|S| ! \tilde{A}(S) Q(\alpha-S) \cdot|S|, \quad \alpha \neq \emptyset,
$$

where $\tilde{A}(S)$ is given by the right hand side of (B.4) ( $\alpha$ replaced by $S$ ).

Proof of Lemma B.2. First we show the following equality by induction in $m$ :

$$
\begin{aligned}
n Q(\alpha)= & \sum_{i=1}^{m-1} \sum_{\substack{S \subseteq \alpha \\
|S|=i}}|S| \cdot|S| ! \tilde{A}(S) Q(\alpha-S) \\
& +\mathbf{S}_{\alpha} n \int_{[0,1]^{m-1}} d s \prod_{i=1}^{m-1}(n-i) \partial_{s_{l}} W\left(s_{1} \ldots s_{i} \mid 1 \ldots i+1\right) \\
& \cdot e^{W\left(s_{1} \ldots s_{m-1} \mid 1 \ldots n\right)},[m=1,2, \ldots, n] \\
& W\left(s_{1} \ldots s_{m-1} \mid 1 \ldots n\right)=\left.W\left(s_{1} \ldots s_{n-1} \mid 1 \ldots n\right)\right|_{s_{m}=s_{m+1}=\ldots=s_{n-1}=1}
\end{aligned}
$$

(i) $m=1$ :

$$
n Q(\alpha)=0+\mathbf{S}_{\alpha} n \exp \sum_{1 \leqq j<k \leqq n} w\left(\alpha_{j} \alpha_{k}\right)
$$

This is true by definition of $Q(\alpha)$.

(ii) $m \rightarrow m+1$ : we have to show:

$$
\begin{aligned}
\mathbf{S}_{\alpha} n & \int_{\lceil 0.1\rceil^{m-1}} d S \prod_{i=1}^{m-1}(n-i) \partial_{s_{i}} W\left(s_{1} \ldots s_{i} \mid 1 \ldots i+1\right) e^{W\left(s_{1} \ldots s_{m}-1 \mid 1 \ldots n\right)} \\
= & \sum_{\substack{S \subset \alpha \\
|S|=m}}|S| \cdot|S| ! \tilde{A}(S) Q(\alpha-S) \\
& \quad+\mathbf{S}_{\alpha} n \int_{[0,1]^{m}} d S \prod_{i=1}^{m}(n-i) \partial_{s_{\imath}} W\left(s_{1} \ldots s_{i} \mid 1 \ldots i+1\right) e^{W\left(s_{1} \ldots s_{m} \mid 1 \ldots n\right)}
\end{aligned}
$$

We use the following equality:

$$
\begin{aligned}
e^{W\left(s_{1} \ldots s_{m-1} \mid 1 \ldots n\right)=} & e^{W\left(s_{1} \ldots s_{m}-1 s_{m}=0 \mid 1 \ldots n\right)} \\
& +\int_{0}^{1} d s_{m} e^{W\left(s_{1} \ldots s_{m} \mid 1 \ldots n\right)} \partial_{s_{m}} W\left(s_{1} \ldots s_{m} \mid 1 \ldots n\right) .
\end{aligned}
$$

We insert (B.10) in the left hand side of (B.9) to obtain a sum of two terms which will be shown to be equal to the two terms on the right hand side of (B.9).

First term:

$$
\begin{gathered}
\mathbf{S}_{\alpha} n \int_{[0,1]^{m-1}} d s \prod_{i=1}^{m-1}(n-i) \partial_{s_{i}} W\left(s_{1} \ldots s_{i} \mid 1 \ldots i+1\right) e^{W\left(s_{1} \ldots s_{m-1} s_{m}=0 \mid 1 \ldots n\right)} \\
\cdot e^{W\left(s_{1} \ldots s_{m-1} s_{m}=0 \mid 1 \ldots n\right)}=e^{W\left(s_{1} \ldots s_{m-1} \mid 1 \ldots m\right)} Q\left(\left\{\alpha_{m+1}, \ldots, \alpha_{n}\right\}\right)
\end{gathered}
$$


With (B.12) we get for (B.11):

first term

$$
\begin{aligned}
= & \mathbf{S}_{\alpha} n(n-1) \ldots(n-m+1) \int_{[0,1]^{m-1}} d s \prod_{i=1}^{m-1} \partial_{s_{i}} W\left(s_{1} \ldots s_{i} \mid 1 \ldots i+1\right) e^{W\left(s_{1} \ldots s_{m}-1 \mid 1 \ldots m\right)} . \\
& \cdot Q\left(\left\{\alpha_{m+1}, \ldots, \alpha_{n}\right\}\right)
\end{aligned}
$$

first term $=\mathbf{S}_{\alpha} n(n-1) \ldots(n-m+1) m \tilde{A}\left(\left\{\alpha_{1}, \ldots, \alpha_{m}\right\}\right) Q\left(\left\{\alpha_{m+1}, \ldots, \alpha_{n}\right\}\right)\left[\mathbf{S}_{\alpha}=\mathbf{S}_{\left\{\alpha_{1}, \ldots, \alpha_{m}\right\}}\right]$. We multiply the right hand side of (B.13) by $\left(\begin{array}{l}n \\ m\end{array}\right)^{-1}$ and sum over $S \subset \alpha,|S|=m$. This
does not change anything and we get:

$$
\begin{aligned}
\text { first term } & =\mathbf{S}_{\alpha} \sum_{\substack{S \subset \alpha \\
|S|=m}} \frac{m !(n-m) !}{n !} m \frac{n !}{(n-m) !} \tilde{A}(S) Q(\alpha-S) \\
& =\sum_{\substack{S \subset \alpha \\
|S|=m}} m m ! \tilde{A}(S) Q(\alpha-S)
\end{aligned}
$$

This is just the first term on the right hand side of (B.9).

Second term:

$$
\begin{gathered}
\mathbf{S}_{\alpha} n \int_{[0,1]^{m}} d s \prod_{i=1}^{m-1}(n-i) \partial_{s_{i}} W\left(s_{1} \ldots s_{i} \mid 1 \ldots i+1\right) \partial_{s_{m}} W\left(s_{1} \ldots s_{m} \mid 1 \ldots n\right) e^{W\left(s_{1} \ldots s_{m} \mid 1 \ldots n\right)} \\
=\mathbf{S}_{\alpha} n \int_{[0,1]^{m}} d s \prod_{i=1}^{m}(n-i) \partial_{s_{i}} W\left(s_{1} \ldots s_{i} \mid 1 \ldots i+1\right) e^{W\left(s_{1} \ldots s_{m} \mid 1 \ldots n\right)}
\end{gathered}
$$

This holds because the integrand is symmetric in $\alpha_{m+1}, \ldots, \alpha_{n}$ and so we can use the fact that everything stands behind the symmetrization operator $\mathbf{S}_{\alpha}$. (B.15) is already the second term on the right hand side of (B.9). End of the proof of the Lemma B.2.

\section{Lemma B.3.}

$$
Q(\alpha)=\frac{1}{n} \sum_{S \subset \alpha}|S| \cdot|S| ! A(S) Q(\alpha-S), \quad \alpha \neq \emptyset .
$$

Proof of Lemma B.3: This proof is similar to the standard proof of KirkwoodSalsburg equations in polymer systems [13].

(i) $Q(\alpha)=\prod_{1 \leqq j<k \leqq n} e^{w\left(\alpha_{j} \alpha_{k}\right)}=\prod_{1 \leqq j<k \leqq n}\left(1+f_{\alpha_{j} \alpha_{k}}\right)$

$$
=\sum_{\mathscr{B}} \prod_{\left(\alpha_{j} \alpha_{k}\right) \in \mathscr{B}} f_{\alpha_{j} \alpha_{k}} \quad[\alpha \neq \emptyset,|\alpha|=n] .
$$

Summation over $\mathscr{B}$ is over all sets of pairs $\left(\alpha_{j}, \alpha_{k}\right)$ with $j, k \in\{1, \ldots, n\}$. We decompose $\mathscr{B}$ in connected pieces and finally arrive at the formula

$$
\begin{gathered}
Q(\alpha)=\sum_{\alpha=\Sigma S_{\varrho}} \prod_{\varrho} \sigma\left(S_{\varrho}\right) ; \quad \sigma\left(S_{\varrho}\right)=\sum_{\mathscr{G}_{S_{\varrho}}\left(x, x_{k}\right) \in \mathscr{G}_{S_{\varrho}}} f_{\alpha_{J} \alpha_{k}}, \\
{\left[\sigma\left(S_{\varrho}\right)=1 \quad \text { for } \quad\left|S_{\varrho}\right|=1\right] .}
\end{gathered}
$$


Summation over $\alpha=\sum_{\varrho} S_{\varrho}$ is over all partitions of $\alpha$ in non-empty mutually disjoint subsets $S_{\varrho}$ with $\bigcup_{\varrho} S_{\varrho}=\alpha$. The sum over $\mathscr{G}_{S_{\varrho}}$ is the same as the analogous one in Eq. (B.3). (B.18) specifies a polymer system living on $\alpha$.

(ii) Given two subsets $Y \subset X \subset \alpha$ with $y \neq \emptyset . X^{\prime}=\Lambda-X$.

We consider

$$
Q\left(X^{\prime}+Y\right)=\sum_{X^{\prime}+Y=\Sigma S} \prod_{S} \sigma(S) .
$$

Given a partition $X^{\prime}+Y=\sum S$ we consider $D=X^{\prime} \cap \bigcup_{S \cap Y \neq \emptyset} S$, where

a) $D$ is an arbitrary subset of $X^{\prime}(D=\emptyset$ is possible).

b) $\sum_{S \cap Y=\emptyset} S$ is a partition of $X^{\prime}-D$.

c) $\sum_{S \cap Y \neq \emptyset} S$ is a partition of $Y+D$ satisfying $S \cap Y \neq \emptyset$ for all $S$ in the partition. It follows that

$$
Q\left(X^{\prime}+Y\right)=\sum_{D \subset X^{\prime}} \sum_{X^{\prime}-D=\Sigma S} \sum_{\substack{Y+D=\sum S^{\prime} \\ S^{\prime} \cap Y \neq \emptyset}} \prod_{S} \sigma(S) \prod_{S^{\prime}} \sigma\left(S^{\prime}\right)
$$

We define

$$
\Phi(Y \mid D)=\sum_{\substack{Y+D=\sum S^{\prime} \\ S^{\prime} \cap Y \neq \emptyset}} \prod_{S^{\prime}} \sigma\left(S^{\prime}\right)
$$

and obtain the equation:

$$
Q\left(X^{\prime}+Y\right)=\sum_{D \subset X^{\prime}} \Phi(Y \mid D) Q\left(X^{\prime}-D\right) .
$$

We choose $X=Y=\alpha_{i}, \alpha_{i}$ some arbitrary element in $\alpha$.

$$
\Phi\left(\alpha_{i} \mid D\right)=\sum_{\substack{\left\{\alpha_{i}\right\}+D=\Sigma S^{\prime} \\ S^{\prime} \ni \alpha_{i}}} \prod_{S^{\prime}} \sigma\left(S^{\prime}\right)=\sigma\left(\alpha_{i}+D\right) .
$$

So we get from Eq.(B.22):

$$
Q(\alpha)=\sum_{\substack{D \subset \alpha \\ D \notin \alpha_{l}}} \sigma\left(\alpha_{i}+D\right) Q\left(\alpha-\left(\alpha_{i}+D\right)\right)=\sum_{\substack{S \subset \alpha \\ S \ni \alpha_{2}}} \sigma(S) Q(\alpha-S) .
$$

We sum Eq. (B.24) over $i=1, \ldots, n$ and divide by $n$ to get:

$$
Q(\alpha)=\frac{1}{n} \sum_{\alpha_{l} \in \alpha} \sum_{\substack{S \subset \alpha \\ S \ni \alpha_{i}}} \sigma(S) Q(\alpha-S)=\frac{1}{n} \sum_{S \subset \alpha}|S| \sigma(S) Q(\alpha-S) .
$$

We compare (B.3) and (B.18) and obtain $\sigma(S)=|S| ! A(S)$.

This proves Lemma B.3. 
Proof of Proposition B.1: Consider the following system of equationr for unknowns $\varphi(S)$ :

$$
\begin{aligned}
& Q(\alpha)=\frac{1}{n} \sum_{S \subset \alpha}|S| \cdot|S| ! \varphi(S) Q(\alpha-S), \quad \alpha \neq \emptyset, \\
& Q(\emptyset)=1, \quad \varphi(\emptyset)=0 .
\end{aligned}
$$

$\varphi$ lives on finite subsets of the set of indices $I$. (B.26) determine the quantities $\varphi(S)$ uniquely:

$$
\frac{1}{n}|\alpha| \cdot|\alpha| ! \varphi(\alpha)=Q(\alpha)-\frac{1}{n} \sum_{\substack{S \subset \alpha \\ S \neq \alpha}}|S| \cdot|S| ! \varphi(S) Q(\alpha-S) .
$$

(B.27) allows a recursive determination of all $\varphi(S)$.

From the Lemmata B.1, B.2 we see that $A(S)$ and $\tilde{A}(S)$ both fulfill the equations (B.26). So they must be identical: $A(S)=\tilde{A}(S)$. This proves Proposition B.1.

\section{Appendix C. Proof of Lemma 5 (The Tree Estimate)}

We wish to estimate quantities of the form

$$
\begin{aligned}
\mathscr{S}(\mu) & \equiv \sum_{\eta} \int_{0}^{1} d s_{1} \ldots d s_{t-1} f(\eta, \mathbf{s}) \prod_{a=1}^{t-1}[\mu(a+1) \mu(\eta(a))] \\
& =\sum_{\eta} \int_{0}^{1} d s_{1} \ldots d s_{t-1} \prod_{a=1}^{t-1}\left[\mu(a+1) s_{a-1} s_{a-2} \ldots s_{\eta(a)} \mu(\eta(a))\right] .
\end{aligned}
$$

The following procedure follows $[11,2]$ closely. Summation over $\eta$ amounts to summing over $\eta(a)=1 \ldots a$ for each $a$. Thus

$$
\mathscr{S}(\mu)=\int_{0}^{1} d s_{1} \ldots d s_{t-1} \prod_{a=1}^{t-1}\left[\sum_{k=1}^{a} \mu(a+1) s_{a-1} s_{a-2} \ldots s_{k} \mu(k)\right] .
$$

Now one inserts a factor

$$
1 \leqq \exp \sum_{b=1}^{t-1} s_{t-1} s_{t-2} \ldots s_{b} \mu(b)
$$

After that the $s$-integrations can be performed in the order $s_{t-1}$ first, then $s_{t-2}$ etc. In each step one uses the inequality

$$
\int_{0}^{1} d s u e^{s u} \leqq e^{u}
$$

Thus, in the first step one estimates

$$
\begin{aligned}
\int_{0}^{1} d s_{t-1} \sum_{k=1}^{t-1} s_{t-2} \ldots s_{k} \mu(k) \leqq & \int d s_{t-1} \sum_{k=1}^{t-1} s_{t-2} \ldots s_{k} \mu(k) \\
& \cdot \exp \left[\sum_{b=1}^{t-1} s_{t-1} \ldots s_{b} \mu(b)\right] \\
\leqq & \exp \left[\mu(t-1)+\sum_{b=1}^{t-2} s_{t-2} \ldots s_{b} \mu(b)\right]
\end{aligned}
$$


Next, the $s_{t-2}$-integral can be estimated in the same way, and so on. As a result after $t-1$ steps one obtains

$$
\mathscr{S}(\mu) \leqq\left\{\prod_{a=1}^{t-1} \mu(a+1)\right\} \exp \sum_{b=1}^{t-1} \mu(b)=\prod_{a=1}^{t-1}\left\{\mu(a+1) e^{\mu(a)}\right\} .
$$

This is the assertion of Lemma 5.

\section{Appendix D. Some Properties of the Lattice Yukawa Potential}

Let $v_{M}(x, y)=\left(-\Delta+M^{2}\right)^{-1}(x y)$ be the Yukawa potential in $v$ dimensions. From its Fourier expansion (cf. eq. (5.5)) we find that

$$
v_{M}(x, 0)=(2 \pi)^{-v} \int d^{v} k\left[2 \sum_{\mu=1}^{v}\left(1-\cos k_{\mu}\right)+M^{2}\right]^{-1} e^{i k x} .
$$

Integration is over $k_{\mu}=-\pi \ldots+\pi$. One inserts the integral representation $x^{-1}=\int_{0}^{\infty} d t e^{-x t}$ for []$^{-1}$. The $k$-integrations can then be performed with the result that

$$
v_{M}(x, 0)=\int_{0}^{\infty} d t e^{-t\left(2 v+M^{2}\right)} \prod_{\mu=1}^{v}\left\{I_{x_{\mu}}(2 t)\right\} .
$$

The modified Bessel functions $I_{n}(x)=I_{-n}(x)$ are positive and monotonically decreasing in $|n|$, for $x \geqq 0$. It follows that

$$
v_{M}(x, 0) \geqq 0 ; \quad v^{2}(x, 0)=v_{M}(x, 0)-v_{M_{1}}(x, 0) \geqq 0 \quad \text { if } \quad M \leqq M_{1}
$$

and $v_{M}$ and $v^{2}$ are monotonically decreasing in the modulus $\left|x_{\mu}\right|$ of each coordinate. (To show monotonicity of $I_{n}(x)$ in $|n|$ for all $x \geqq 0$ one computes $I_{n}(x)-I_{n+1}(x)$ from the integral representation, formula 8.431 of [14], writing $z e^{z t}$ $=(\partial / \partial t) e^{z t}$ and reabsorbing $\partial / \partial t$ by a partial integration. The result is an integral representation with a manifestly positive integrand.)

\section{References}

1. Ruelle, D. : Statistical mechanics. New York: Benjamin 1969, and references to earlier work given there

2. Brydges, D., Federbush, P.: J. Math. Phys. 19, 2064 (1978)

3. Polyakov, A.M. : Nucl. Phys. B120, 429 (1977)

Drell, S.D., Quinn, H.R., Svetitsky, B., Weinstein, M.: Phys. Rev. D19, 619 (1979)

4. Banks, T., Myerson, R., Kogut, J.: Nucl. Phys. B129, 493 (1977)

5. Göpfert, M., Mack, G.: Submitted to Commun. Math. Phys.

6. Brydges, D.: Commun. Math. Phys. 58, 313 (1978); see also Brydges, D., Federbush, P.: Commun. Math. Phys. 73, 197 (1980)

7. Wilson, K.: Phys. Rev. D2, 1473 (1970)

Kadanoff, L.P.: Physics 2, 263 (1965)

Kogut, J., Wilson, K.: Phys. Rep. 12C, 76 (1974)

Gawedzki, K., Kupiainen, A. : Commun. Math. Phys. 77, 31 (1980) 
8. Fröhlich, J., Spencer, T.: Phase diagrams and critical properties of (classical) Coulomb systems. Erice lectures 1980

9. Villain, J.: J. Phys. (Paris) 36, 581 (1975)

10. Huang, K.: Statistical mechanics. New York: Wiley 1963

11. Glimm, J., Jaffe, A., Spencer, T.: Ann. Math. 100, 585 (1974)

12. Ukawa, A., Windey, P., Guth, A.H.: Phys. Rev. D21, 1013 (1980)

Müller, V.F., Rühl, W.: Discrete field variables, the Coulomb gas, and low temperature behaviour. Preprint Kaiserslautern (January 1981)

13. Gruber, Ch., Kunz, A.: Commun. Math. Phys. 22, 133 (1971)

14. Gradshteyn, I.S., Ryzhik, I.M.: Table of integrals, series and products. New York: Academic Press 1965

Communicated by R. Haag

Received April 13, 1981 